\title{
Multicast Capacity of Wireless Ad Hoc Networks
}

\author{
Xiang-Yang Li, Associate Member, IEEE,
}

\begin{abstract}
We study the capacity of a large-scale random wireless network for multicast. Assume that $n$ wireless nodes are uniformly randomly deployed in a square region with sidelength $a$ and all nodes have the uniform transmission range $r$ and uniform interference range $R>r$. We further assume that each wireless node can transmit (or receive) at $W$ bits/second over a common wireless channel. For each node $v_{i}$, we randomly and independently pick $k-1$ points $p_{i, j}(1 \leq j \leq k-1)$ from the square, and then multicast data to the nearest node for each $p_{i, j}$. The aggregated multicast capacity is defined as the total data rate of all multicast sessions in the network. We derive matching asymptotic upper bounds and lower bounds on multicast capacity of random wireless networks. Under protocol interference model, when $a^{2} / r^{2}=O(n / \log (n))$, we show that the total multicast capacity is $\Theta\left(\sqrt{\frac{n}{\log n}} \cdot \frac{W}{\sqrt{k}}\right)$ when $k=O\left(\frac{n}{\log n}\right)$; the total multicast capacity is $\Theta(W)$ when $k=\Omega\left(\frac{n}{\log n}\right)$. Our bounds unify the previous capacity bounds on unicast (when $k=2$ ) by Gupta and Kumar [6] and broadcast (when $k=n$ ) in [9], [20]. We also study the capacity of group-multicast for wireless networks where for each source node, we randomly select $k-1$ groups of nodes as receivers and the nodes in each group are within a constant hops from the group leader. The same asymptotic upper bounds and lower bounds still hold. We also extend our capacity bounds to $d$-dimensional networks.
\end{abstract}

Index Terms-Wireless ad hoc networks, capacity, multicast, broadcast, unicast, scheduling, optimization, VC dimension.

\section{INTRODUCTION}

In many applications, e.g., wireless sensor networks, we often need estimate the achievable throughput of the deployed network. The main purpose of this paper is to study the asymptotic capacity of large scale random wireless networks when we choose the best protocols for all layers. As in the literature, we will mainly consider one type of networks, large scale random networks, where a large number of nodes are uniformly randomly placed in the deployment region. We will study the capacity of a given wireless network where the nodes positions are randomly given a priori, and how the capacity of wireless networks scale with the number of nodes in the networks (when given a fixed deployment region), or scale with the size of the deployment region (when given a fixed deployment density). We assume that a set of $n$ wireless nodes $V=\left\{v_{1}, v_{2}, \cdots, v_{n}\right\}$ are randomly distributed (with uniform distribution) in a square region with a side-length $a$ and all nodes have the same transmission range $r$. For majority results presented in this paper, we assume that the deployment

Department of Computer Science, Illinois Institute of Technology, Chicago, IL. Part of the work was done when the author visited Microsoft Research Asia, BeiJing, China. Email: xli@cs.iit.edu. The preliminary result [15] was published at ACM MobiCom 2007. The research of the author was partially supported by National Basic Research Program of China (973 Program) under grant No. 2006CB30300, the National High Technology Research and Development Program of China (863 Program) under grant No. 2007AA01Z180, the RGC under Grant HKBU 2104/06E and CERG under Grant PolyU-5232/07E. region $a$ and the transmission range $r$ are selected such that the resulted network will be connected with high probability (w.h.p.). The results derived under this model also imply the same results for the dense model, when $n$ nodes are distributed in a fixed region (such as a unit square by a proper scaling) and the uniform transmission range of all nodes are selected as the critical transmission range (CTR) to get a connected network w.h.p..

In this paper, we will concentrate on the multicast capacity of a random wireless network, which generalizes both the unicast capacity [6] and broadcast capacity [9], [20] for random networks. Assume that a subset $\mathcal{S} \subseteq V$ of $n_{s}=|\mathcal{S}|$ nodes will serve as the source nodes of $n_{s}$ multicast sessions. Each node $v_{i} \in \mathcal{S}$ randomly and independently chooses $n_{d}=k-1$ points $P_{i}=\left\{p_{i, j} \mid 1 \leq j \leq k-1\right\}$ in the square. For each point $p_{i, j}$, let $v_{i, j}$ be the node from $V$ that is the closest to $p_{i, j}$. Then node $v_{i}$ will send data to these $k-1$ nodes $U_{i}=\left\{v_{i, j} \mid 1 \leq j \leq k-1\right\}$ at an arbitrary data rate $\vartheta_{i}$. The aggregated multicast capacity with $\mathcal{S}$ as roots for a network is defined as $\Lambda_{k, \mathcal{S}}(n)=\sum_{v_{i} \in \mathcal{S}} \vartheta_{i}$ when there is a schedule of transmissions such that all multicast flows will be received by their destination nodes successfully within a finite delay.

Due to spatial separation, several wireless nodes can transmit simultaneously provided that these transmissions will not cause destructive wireless interferences to any of the transmissions. To describe when a transmission is received successfully by its intended recipient, we will allow one possible model for a successful one-hop reception: protocol model where each node $v \in V$ has a fixed constant interference range $R>r$. A node $u$ can successfully receive a transmission from another node $v$ iff there is no other node $w$ such that $\|w-u\| \leq R$ and node $w$ is transmitting simultaneously with node $v$. Here $\|w-u\|$ is the Euclidean distance between $w$ and $u$.

We assume the following simple wireless channel model as in the literature: each wireless node can transmit at $W$ bits/second over a common wireless channel. For presentation simplicity, we assume that there is only one channel in the wireless networks. As always, we assume that the packets are sent from node to node in a multi-hop manner until they reach their final destinations. The packets could be buffered at intermediate nodes while awaiting for transmission. The only operation by relay node is to receive, store, and forward packets. In this paper, we assume that the buffer is large enough so packets will not get dropped by any intermediate node. We leave it as future work to study the scenario when the buffers of intermediate nodes are bounded by some values. For most of the results presented here, the delay of the routing is not considered, i.e., the delay in the worst case could be arbitrarily large for some results.

Our Main Contributions: In this paper we propose two regimes for multicast capacity in terms of $k$. We derive match- 
ing analytical upper bounds and lower bounds on multicast capacity of a random wireless network. Assume that the sidelength $a$ of the deployment square and the transmission range $r$ are selected such that the network is connected w.h.p., i.e., $\frac{a}{r}=\Theta\left(\sqrt{\frac{n}{\log n}}\right)$. We show that the aggregated multicast capacity of $n$ multicast sessions is

$$
\Lambda_{k}(n)= \begin{cases}\Theta\left(\sqrt{\frac{n}{\log n}} \cdot \frac{W}{\sqrt{k}}\right) & \text { when } k=O\left(\frac{n}{\log n}\right), \\ \Theta(W) & \text { when } k=\Omega\left(\frac{n}{\log n}\right)\end{cases}
$$

Our bounds unify the capacity bounds on unicast (when $k=2$ ) by Gupta and Kumar [6] and the capacity bounds on broadcast (when $k=n$ ) in [9], [20]. More generally, we prove that the aggregated multicast capacity of $n_{s}$ random multicast sessions has the same asymptotic upper-bound as formula (1), and has the same asymptotic lower-bound as formula (1) whenever $n_{s} \geq \Theta\left(\log k \cdot \sqrt{\frac{n \log n}{k}}\right)$. In addition, the per-flow capacity $\lambda_{k}(n)$ of $n$ multicast sessions is

$$
\lambda_{k}(n)= \begin{cases}\Theta\left(\sqrt{\frac{1}{n \log n}} \cdot \frac{W}{\sqrt{k}}\right) & \text { when } k=O\left(\frac{n}{\log n}\right), \\ \Theta\left(\frac{W}{n}\right) & \text { when } k=\Omega\left(\frac{n}{\log n}\right)\end{cases}
$$

The preceding capacity bounds are implied by a more general result for the following network setting: there are $n_{s}$ multicast sessions, each with $k-1$ receivers from $V$, and the transmission range $r$ and side-length $a$ of the deployment square satisfying that the resultant random network is connected w.h.p. Generally, when $n_{s} \geq \Omega\left(\log k \cdot \sqrt{\frac{n \log n}{k}}\right)$, we prove that the aggregated capacity of $n_{s}$ multicast sessions is

$$
\Lambda_{k}(n)= \begin{cases}\Theta\left(\frac{a}{r} \cdot \frac{W}{\sqrt{k}}\right) & \text { when } k=O\left(\frac{a^{2}}{r^{2}}\right) \\ \Theta(W) & \text { when } k=\Omega\left(\frac{a^{2}}{r^{2}}\right)\end{cases}
$$

We also study the multicast capacity for group-multicast where, for each source node, we randomly select $k-1$ groups of nodes as receivers and the nodes in each group are within a constant number of hops from the group leader (who are nodes closest to $k-1$ randomly chosen points). We show that the asymptotic multicast capacity is still $\Theta\left(\sqrt{\frac{n}{\log n}} \cdot \frac{W}{\sqrt{k}}\right)$ when $k=O\left(\frac{n}{\log n}\right)$; and is $\Theta(W)$ when $k=\Omega\left(\frac{n}{\log n}\right)$. We also extend our results to $d$-dimensional networks. Our techniques also imply a different proof for the unicast capacity [6] for 2dimensional networks without using mapping to networks on sphere surface (was used in [6]). Our new techniques enable us deriving capacity bounds for $d$-dimensional networks also.

The rest of the paper is organized as follows. In Section II we discuss the network and channel model and review the capacity definition. We briefly discuss the proof techniques that we use to analyze the capacity upper-bound in Section III. In Section IV, we first present some upper-bounds on multicast capacity for random networks. In Section V, we then present an efficient method for multicast and prove that the capacity achieved by this method asymptotically matches the upperbounds derived before. In Section VI, we study the multicast capacity bounds for group-multicast and the multicast capacity bounds for $d$-dimensional networks. We review the related results on network capacities in Section VII and conclude the paper in Section VIII.

\section{NeTWORK MODEL}

The capacity of random wireless networks was first studied in a landmark seminal work by Gupta and Kumar [6]. There are different approaches to increase the network throughput, such as reducing the interference, the scheduling on the MAC layer, route selection on the routing layer, channel assignment if multi-channels are available, and power control on the physical layer. In this section, we first introduce our network system model, discuss in detail the interference models we will use, then define the problem that we will study.

In this paper, we assume that there is a set $V=$ $\left\{v_{1}, v_{2}, \cdots, v_{n}\right\}$ of $n$ communication terminals deployed in a region $\Omega$. We mainly focus on the scenario when $\Omega$ is a square with side length $a$. Every wireless node has a transmission range $r$ such that two nodes $u$ and $v$ can communicate directly if $\|u-v\| \leq r$ and there is no other interference. The complete communication graph is a undirected graph $G=(V, E)$, where $E$ is the set of possible communication links $u v$ with $\|u-v\| \leq r$. The majority results presented in this paper rely on the ratio $\frac{a}{r}$, where either $a$ or $r$ or both could be a function of $n$. In this paper, we mainly assume that the transmission range $r$ is a constant. Under this assumption, the side length $a$ of the deployment square region $\Omega$ will be a function of $n$.

To schedule two links at the same time slot, we must ensure that the schedule will avoid interference. Several different interference models have been used to model the interferences in wireless networks. In this paper, we will mainly focus on the protocol interference model. We assume that each node $v_{i}$ has a constant interference range $R$. Here any node $v_{j}$ will be interfered by the signal from $v_{k}$ if $\left\|v_{k}-v_{j}\right\| \leq R$ and node $v_{k}$ is sending signal to some node other than $v_{j}$. In this paper, we always assume that the interference range $R$ is within a small constant factor of the transmission range $r$, i.e., $\beta_{0} \cdot r \leq R \leq \beta \cdot r$ for constants $\beta \geq \beta_{0}>1$.

Capacity Definition: We assume that each node $v_{i}$ could serve as the source node for some multicast. Assume that a subset $\mathcal{S} \subseteq V$ of $n_{s}=|\mathcal{S}|$ nodes will serve as the source nodes of $n_{s}$ multicast sessions. For each node $v_{i}$, we randomly and independently choose a set $P_{i}$ of $n_{d}=k-1$ points $P_{i}=\left\{p_{i, j} \mid 1 \leq j \leq k-1\right\}$ in the deployment square. For each point $p_{i, j}$, let $v_{i, j}$ be the node from $V$ that is the closest to $p_{i, j}$ (arbitrarily choose one if there are multiple nodes with the same shortest distance). Nodes $U_{i}=\left\{v_{i, j} \mid 1 \leq j \leq k-1\right\}$ will be the destination nodes of multicast from $v_{i}$. Then node $v_{i}$ will send data to these $k-1$ nodes $U_{i}$ at an arbitrary data rate $\vartheta_{i}$. Notice that when the receivers are far away from the source node, we need multiple intermediate nodes to relay the data for $v_{i}$. The only operation the intermediate nodes can do is to store and then forward the data packets. No any other operation (such as successive interference canceling and network coding) is allowed. Thus, a throughput optimal routing will use a spanning tree rooted at the source and span all receivers. Thus, we only consider spanning tree as routing structure for multicast.

Given the set $\mathcal{S}$ of $n_{s}=|\mathcal{S}|$ source nodes, let $\lambda_{\mathcal{S}}=$ $\left(\vartheta_{i_{1}}, \vartheta_{i_{2}}, \cdots, \vartheta_{i_{n_{s}-1}}, \vartheta_{n_{s}}\right)$ be the rate vector of the multicast data rate of all $n_{s}$ multicast sessions. Here $\vartheta_{i_{j}}$ is the data rate 
of node $v_{i_{j}} \in \mathcal{S}$, for $1 \leq j \leq n_{s}$. When given a fixed network $G=(V, E)$, where the node positions of all nodes $V$, the set of receivers $U_{i}$ for each source node $v_{i}$, and the multicast data rate $\vartheta_{i}$ for each source node $v_{i}$ are all fixed, we first define what is a feasible rate vector $\vartheta$ for the network $G$.

Definition 1 (Feasible Rate Vector): Given set $\mathcal{S}$ of $n_{s}$ source nodes, a multicast rate vector $\vartheta_{\mathcal{S}}$ bits/sec is feasible if there is a spatial and temporal scheme for scheduling transmissions such that by operating the network in a multihop fashion and buffering at intermediate nodes when awaiting transmission, every node $v_{i}$ can send $\vartheta_{i}$ bits/sec average to its chosen $k-1$ destination nodes. That is, there is a $T<\infty$ such that in every time interval (with unit seconds) $[(i-1) \cdot T, i \cdot T]$, every node $v_{i} \in \mathcal{S}$ can send $T \cdot \vartheta_{i}$ bits to each of its corresponding $k-1$ receivers $U_{i}$.

The total multicast throughput capacity of such feasible rate vector for multicast is defined as $\Lambda_{k, \mathcal{S}}(n)=\sum_{v_{i} \in \mathcal{S}} \vartheta_{i}$. The average per-flow multicast throughput capacity is $\lambda_{k, \mathcal{S}}^{a}(n)=$ $\frac{\sum_{v_{i} \in \mathcal{S}} \vartheta_{i}}{n_{s}}$. The minimum per-flow multicast throughput capacity is $\lambda_{k, \mathcal{S}}(n)=\min _{v_{i} \in \mathcal{S}} \vartheta_{i}$, where $k$ is the total number of nodes in each multicast session, including the source node. When $\mathcal{S}$ is clear from the context, we drop $\mathcal{S}$ from our notations. When we mention per-flow multicast capacity, hereafter we mean the minimum per flow multicast capacity, if not explained otherwise.

Definition 2 (Throughput Capacity): An aggregated multicast throughput $\Lambda_{k}(n)$ bits/sec is feasible for $n_{s}$ multicast sessions (each session with $k$ terminals) if there is a rate vector $\lambda_{\mathcal{S}}=\left(\vartheta_{i_{1}}, \vartheta_{i_{2}}, \cdots, \vartheta_{i_{n_{s}-1}}, \vartheta_{i_{n_{s}}}\right)$ that is feasible and $\Lambda_{k}(n)=\sum_{v_{i} \in \mathcal{S}} \vartheta_{i}$. Similarly, we say $\lambda_{k}(n)=\min _{v_{i} \in \mathcal{S}} \vartheta_{i}$ is a feasible per-flow multicast throughput capacity.

Definition 3 (Capacity of Random Networks): The aggregated multicast capacity of a class of random networks is of order $\Theta(g(n))$ bits/sec if there are deterministic constants $c>0$ and $c<c^{\prime}<+\infty$ such that

$$
\begin{aligned}
\lim _{n \rightarrow \infty} \operatorname{Pr}\left(\Lambda_{k}(n)=c g(n) \text { is feasible }\right) & =1 \\
\liminf _{n \rightarrow \infty} \operatorname{Pr}\left(\Lambda_{k}(n)=c^{\prime} g(n) \text { is feasible }\right) & <1
\end{aligned}
$$

We can similarly define the per-flow capacity for random networks. Here the probability is computed using all possible connected random networks formed by $n$ nodes distributed in a square with side-length $a$.

Useful Known Results: Throughput this paper, we will repeatedly use the following results:

Lemma 1 (Chebyshev's Inequality): For a variable $X$,

$$
\operatorname{Pr}(|X-\mu| \geq A) \leq \frac{\operatorname{Var}(X)}{A^{2}},
$$

where $A>0, \mu=E(X), \operatorname{Var}(X)$ is the variance of $X$.

Lemma 2 (Binomial Distribution): Consider $n$ independent variables $X_{i} \in\{0,1\}$ with $p=\operatorname{Pr}\left(X_{i}=1\right)$. Let $X=$ $\sum_{i=1}^{n} X_{i}$. Then

$$
\begin{gathered}
\operatorname{Pr}(X \leq \xi) \leq e^{\frac{-2(n \cdot p-\xi)^{2}}{n}}, \quad \text { when } 0<\xi \leq n \cdot p . \\
\operatorname{Pr}(X>\xi)<\frac{\xi(1-p)}{(\xi-n \cdot p)^{2}}, \quad \text { when } \xi>n \cdot p .
\end{gathered}
$$

We will also use the uniform convergence in the weak law of large numbers. We recall the following definitions by Vapnik and Chervonenkis [21]. Let $\mathcal{U}$ be the input space. Let $\mathcal{C}$ be a family of subsets of $\mathcal{U}$. A finite set $S$ (called sample in machine learning) is shattered by $\mathcal{C}$, if for every subset $B$ of $S$, there exists a set $A \in \mathcal{C}$ such that $A \cap S=B$. The VC-dimension of $\mathcal{C}$, denoted by $\operatorname{VC}-\mathrm{d}(\mathcal{C})$, is defined as the maximum value $d$ such that there exists a set $S$ with cardinality $d$ that can be shattered by $\mathcal{C}$. For sets of finite VC-dimension, one has uniform convergence in the weak law of large numbers:

Theorem 3 (The Vapnik-Chervonenkis Theorem): If $\mathcal{C}$ is a set of finite VC-dimension $\mathrm{VC}-\mathrm{d}(\mathcal{C})$, and $\left\{X_{i} \mid i=\right.$ $1,2 \cdots, N\}$ is a sequence of i.i.d. random variables with common probability distribution $P$, then for every $\epsilon, \delta>0$,

$$
\operatorname{Pr}\left(\sup _{A \in \mathcal{C}}\left|\frac{\sum_{i=1}^{N} I\left(X_{i} \in A\right)}{N}-P(A)\right| \leq \epsilon\right)>1-\delta
$$

whenever

$$
N>\max \left\{\frac{8 \cdot \operatorname{VC}-\mathrm{d}(\mathcal{C})}{\epsilon} \cdot \log \frac{13}{\epsilon}, \frac{4}{\epsilon} \log \frac{2}{\delta}\right\} .
$$

Here $I\left(X_{i} \in A\right)$ takes value 1 if $X_{i} \in A$ and 0 otherwise.

Notations: Throughput this paper, for a continuous region $\Omega$, we use $|\Omega|$ to denote its area; for a discrete set $S$, we use $|S|$ to denote its cardinality; for a tree $T$, we use $\|T\|$ to denote its total Euclidean edge lengths; $x \rightarrow \infty$ denotes that variable $x$ takes value to infinity. We use $D(x, b)$ to denote a disk centered at a point $x$ with radius $b$.

\section{General Techniques For UpPer-Bounds}

\section{A. Hop Counts}

In previous studies [6], [18] of capacity of random networks, a common approach is to analyze the expected number of hops $H(b)$ a bit $b$ has to travel and the total number $S$ of simultaneous transmissions $\left(S=O\left(\frac{a^{2}}{r^{2}}\right)\right.$ by simple area argument) possible in the system. If each source node generates data at rate $\vartheta$, the number of bits generated by these $n_{s}$ sources in time interval $T$ is simply $\vartheta T n_{s}$. Thus, the total number of transmissions of all bits to their destinations is $\vartheta T n_{s} H(b)$ almost surely. Thus, we have $\vartheta T n_{s} H(b) \leq T \cdot S$. This implies that $\vartheta=O\left(\frac{a^{2}}{r^{2}} \cdot \frac{1}{n_{s} H(b)}\right)=O\left(\frac{n}{\log n} \cdot \frac{1}{n_{s} H(b)}\right)$. In [6], for unicast, Gupta and Kumar essentially used $\Theta\left(\frac{1}{r}\right)$ (assumed $a=1$ ) as estimation of $H(b)$ and derived $\Theta\left(\frac{W}{n_{s} \cdot r}\right)$ as per-flow capacity upper-bound. In [18], for multicast, Shakkottai et al. essentially used $H(b)=\Theta\left(\frac{\sqrt{k}}{r}\right)$ (assumed $a=1$ ) to derive $O\left(\frac{W}{n_{s} \cdot \sqrt{k} \cdot r}\right)=O\left(\frac{\sqrt{n}}{n_{s} \sqrt{k \log n}}\right)$ as per-flow capacity upperbound since $r=\Theta(\sqrt{\log n / n})$. Although this traditional technique is valid and convenient for studying the asymptotic unicast capacity and the multicast capacity with some special configurations $\left(k=n^{1-\epsilon}\right.$ for some $\left.0<\epsilon<1\right)$ [18], this may produce a pessimistic or even erroneous upper-bound for asymptotic multicast capacity in a general setting studied in this paper. For example, when $k=n$ (i.e., broadcast), formula $O\left(\frac{\sqrt{n}}{n_{s} \sqrt{k} \log n}\right)$ only produces an upper-bound $O\left(\frac{1}{n_{s} \sqrt{\log n}}\right)$, which is asymptotically smaller than the achievable per-flow broadcast capacity $\Theta\left(\frac{1}{n_{s}}\right)$ implied in [9], [10], [20]. The reason 
for this discrepancy is that for multicast we can utilize wireless broadcast nature to reduce transmissions. To address the above challenges and discrepancies, we use two new approaches to analyze the upper-bound of multicast capacity.

\section{B. Shaded Area of Transmissions}

The area argument essentially works as follows. When we multicast from one source node $v_{i}$ to all its $k-1$ receivers $U_{i}$, all nodes lying inside the interference region of any transmitting node for this multicast session cannot receive data from other nodes simultaneously. Given a transmitting node $v_{i}$, we call the interference region $D\left(v_{i}, R\right)$ of node $v_{i}$ the shaded region of node $v_{i}$, where no node inside $D\left(v_{i}, R\right)$ can receive data from nodes other than $v_{i}$ when $v_{i}$ is transmitting. See Fig 1(a) for illustration. The union of all interference disks of a multicast forms the shaded region of that multicast.

For any node $u$, let $t_{i}(u)$ be the time-intervals that node $u$ will transmit data for multicast tree $T_{i}$. Thus, a multicast tree will claim a number of cylinders $\left(D(u, r) \times t_{i}(u)\right.$ for internal node $u$ in $T$ ) in the space-time dimension $\mathcal{R}^{2} \times \mathcal{T}$, where $D(u, r)$ denotes the transmission disk of node $u, \mathcal{T}$ is the scheduling period. Thus, given a multicast tree $T_{i}$ for multicast originated from $v_{i}$, the pairs of $\left(D(u, r), t_{i}(u)\right)$ [i.e., transmission disk $D(u, r)$ that will be used for multicast originated at $v_{i}$ during the transmission time-interval $\left.t_{i}(u)\right]$ claimed by this multicast should be disjoint from the pairs claimed by other multicast sessions. Let $A_{i}$ be the area of the union region of all transmitting nodes for multicast session of node $v_{i}$, and $\vartheta_{i}$ be the data rate of this multicast session. Then obviously $\sum_{i=1}^{n} \vartheta_{i} \cdot A_{i} \leq W \cdot \Phi$ where $\Phi$ is the total area of the region covered by the transmitting disks of all nodes. Thus, it is not difficult to prove the following lemma:

Lemma 4: For any task, such as multicast, let $A_{i}$ be the area of the union region of all transmission disks of all transmitting nodes for task initiated by node $v_{i}$. If $A_{i}$ is at least $\wp$ w.h.p., for every node $v_{i}$ in $\mathcal{S}$, then, w.h.p., the aggregated capacity for this task in a random network deployed in a region with area $\Phi$ is at most $\frac{\Phi \cdot W}{\wp}$. Similarly, when $\sum_{v_{i} \in \mathcal{S}} A_{i}$ is at least $\mathcal{A}$ w.h.p., the per-flow capacity $\lambda_{k}(n)$ is at most $\frac{\Phi \cdot W}{\mathcal{A}}$ w.h.p..

Notice $\Lambda_{k}(n) \cdot \wp=\sum_{i=1}^{n} \vartheta_{i} \cdot \wp \leq_{\text {w.h.p. }} \sum_{i=1}^{n} \vartheta_{i} \cdot A_{i} \leq W \cdot \Phi$; $\lambda_{k}(n) \cdot \mathcal{A} \leq_{\text {w.h.p. }} \lambda_{k}(n) \sum_{i=1}^{n} A_{i} \leq \sum_{i=1}^{n} \vartheta_{i} \cdot A_{i} \leq W \cdot \Phi$.

\section{Data Copies}

When we multicast from one source node $v_{i}$ to all its $k-1$ receivers $U_{i}$, some nodes (not relay nodes and not receiver) may overhear and get a copy of the data. Observe that when a relay node $v$ is transmitting at time-slot $t$, any node $w$ inside the interference region $D(v, R)$ cannot receive data simultaneously from any other transmitting node due to interference. In this case, we say that node $w$ also "receives" the data at time-slot $t$. Here node $w$ (not next-hop relay node) may not receive the data physically. For the purpose of analysis, when a node $v$ is transmitting at time $t$, all nodes in $D(v, r)$ will be virtually allocated a copy of the data at time $t$. See Fig 1(a) for illustration: all nodes inside the shaded region will be allocated a copy of data. For interference model considered here, Lemma 5 in [23] implies that at any time-slot, every node $w$ is allocated at most $\psi=\left\lceil 2 \pi / \arcsin \left(\frac{\beta_{0}-1}{2 \beta_{0}}\right)\right\rceil$ copies of data (from possibly different multicast sessions).

Generally, for a multicast session with source node $v_{i}$, assume that $C_{i}$ nodes will be allocated a copy of its data when the $k-1$ receivers are randomly selected. Obviously, $\sum_{v_{i} \in \mathcal{S}} \vartheta_{i} \cdot C_{i} \leq n \cdot \psi W$ : the left-hand side is the total copies allocated by all sessions, the right-hand side is total data that can be allocated to all nodes. Further assume that $C_{i} \geq C$ almost surely, i.e., $\operatorname{Pr}\left(C_{i} \geq C\right) \rightarrow 1$ as $n$ or $k$ goes to infinity. Then the total multicast capacity satisfies, almost surely, $\Lambda_{k}(n)=\sum_{v_{i} \in \mathcal{S}} \vartheta_{i} \leq \frac{n \cdot \psi W}{C}$. Clearly, $C \geq k$. The following lemma is straightforward.

Lemma 5: For any task, such as multicast, let $X_{i}$ be the number of nodes fall inside shaded region for the task initiated by source $v_{i}$. Assume that $X_{i}$ is at least $\mathcal{N}$ w.h.p. for every source node $v_{i}$. Then, w.h.p., the aggregated capacity by all nodes in a random network of $n$ nodes is at most $\frac{n \cdot \psi W}{\mathcal{N}}$. Moreover, if $\sum_{v_{i} \in \mathcal{S}} X_{i} \geq \mathcal{X}$ w.h.p. for a set $\mathcal{S}$ of sources, then the per-flow capacity is at most $\frac{n \cdot \psi W}{\mathcal{X}}$.

In our proofs, we will utilize these two technical lemmas to give upper-bound on the capacity of a random network for a task that will be performed by each node of the network, such as multicast. Notice that the above lemmas require us to find largest $\wp$ such that $\operatorname{Pr}(\mathcal{A} \geq \wp) \rightarrow 1$, or the largest $\mathcal{N}$ such that $\operatorname{Pr}(\mathcal{X} \geq \mathcal{N}) \rightarrow 1$.

\section{UpPer BOUNDS ON MULTiCASt CAPACiTy FOR RANDOM NETWORKS}

To our surprise, we find that the multicast capacity of a random network where each multicast session has $k-1$ receivers has two regimes: when the number of receivers $k-1$ is over some threshold, multicast capacity is asymptotically same as the broadcast capacity; otherwise, the multicast capacity decreases linearly over $\frac{1}{\sqrt{k}}$. In the next subsections, we will provide upper-bounds for each case separately.

\section{A. The upper-bound on $\frac{a}{r}$ for Connectivity}

We assume that $n$ wireless nodes $V$ with transmission range $r$ are randomly and uniformly distributed in a square region with side length $a$. We first study the asymptotic bound on $a / r$ such that the resulted network $G=(V, E)$ is connected with probability going to 1 as $n$ goes to infinity. Notice that for a set of nodes, the CTR (critical transmission range) for connectivity is always the length of the longest edge of the Euclidean minimum spanning tree (EMST) of this set of nodes [7], [16], [17]. Thus, studying the CTR for connectivity is equivalent to studying the longest edge of the EMST of a set $V$ of nodes when $V$ follows a certain distribution such as Poisson distribution or random uniform distribution.

Assume $n$ points are distributed uniformly at random in the 2-dimensional square with side length $a$. Let $M_{n, a}$ be the random variable denoting the length of the longest edge of EMST built on this set of $n$ nodes. It was proved in [16] that, for any real number $\beta, \lim _{n \rightarrow \infty} \operatorname{Pr}\left(n \pi \cdot M_{n, 1}^{2}-\log n \leq \beta\right)=\frac{1}{e^{e^{-\beta}}}$. Then a simple scaling shows that

$$
\lim _{n \rightarrow \infty} \operatorname{Pr}\left(n \pi \cdot\left(\frac{M_{n, a}}{a}\right)^{2}-\log n \leq \beta\right)=\frac{1}{e^{e^{-\beta}}} .
$$


for any real number $\beta$. Thus, the longest edge length $M_{n, a}$, of EMST built on $n$ points distributed in a square with sidelength $a$, with probability $\frac{1}{e^{e-\beta}}$, is at most $\sqrt{\frac{\log n+\beta}{n \pi}} \cdot a$. Thus, we have

Theorem 6: Assume that $n$ nodes, each with transmission range $r$, are randomly uniformly deployed in a square region of side length $a$. When $\frac{a}{r} \leq \sqrt{\frac{n \pi}{\log n+\beta}}$ for $\beta \rightarrow \infty$, the resulted network $G=(V, E)$ is connected with probability at least $\frac{1}{e^{e^{-\beta}}}$. When $\frac{a}{r} \geq \sqrt{\frac{n \pi}{\log n+\beta}}$ for $\beta \rightarrow-\infty$ (e.g., $\beta=-\ln \ln n)$, the resulted network $G=(V, E)$ is not connected with probability at least $1-\frac{1}{e^{-\beta}}(e . g ., 1-1 / n)$.

If $a=r \sqrt{\frac{n}{\log n}}$, the network is connected with probability at least $e^{-1 / n^{(\pi-1) / \ln 2}}>1-1 / n^{(\pi-1) / \ln 2}>1-1 / n^{2}$.

\section{B. When $k=O\left(a^{2} / r^{2}\right)$}

We first study the multicast capacity when the number of receivers is at most $O\left(a^{2} / r^{2}\right)$. We will present upper bound of the total multicast capacity. A trivial upper bound for total multicast capacity is $W \cdot n$ since there are $n$ source nodes and each source node can only send $W$ bits/sec. A refined upper bound is $\Lambda_{k}(n) \leq \frac{n \cdot W}{k}$ which is derived from the perspective of recipients: (1) each node can receive at most $W$ bits/sec, and (2) among received data by all nodes, any data from any source node will have at least $k$ copies (one copy at each of the $k-1$ receivers and one copy at the source node). From Lemma 5, for a multicast tree $T_{i}$ spanning source node $v_{i}$ and $k-1$ receivers $U_{i}$, we would like to know a lower bound on the number of internal nodes used in $T_{i}$. To analyze this value, we first study the asymptotic lower bound of the Euclidean length $\left\|T_{i}\right\|$ of a multicast tree $T_{i}$.

Lemma 7: [3] Given any $k$ nodes $U$, any multicast tree spanning these $k$ nodes (may be using some additional relay nodes) will have an Euclidean length at least $\varrho \cdot\|E M S T(U)\|$, where $\varrho=\frac{\sqrt{3}}{2}$ and $\operatorname{EMST}(U)$ is the EMST spanning $U$.

Based on Lemma 7 , to get a lower bound on $\left\|T_{i}\right\|$ of any multicast tree $T_{i}$, we need study the length of EMST spanning these $k$ random nodes, which was established in [19].

Lemma 8: [19] The total edge length of the EMST of $n$ nodes randomly and uniformly distributed in a $d$-dimensional cube of side-length $a$ is asymptotic to $\tau(d) \cdot n^{\frac{d-1}{d}} \cdot a$, where $\tau(d)$ is a constant depending only on the dimension $d$.

Thus, based on Lemma 7 and Lemma 8, we have

Lemma 9: The total edge length, denoted by $\left\|T_{i}\right\|$, of any multicast tree $T_{i}$ spanning $k$ nodes that are randomly placed in a square of side-length $a$ almost surely is at least,

$$
\tau \cdot \sqrt{k} \cdot a
$$

where $\tau=\varrho \cdot \tau(2)$, when $k \rightarrow \infty$.

Let $X=\|\operatorname{EMST}(U)\|$ for a set of $k$ randomly selected nodes $U$ in a square of side-length $a$. It was shown in [19] that $\operatorname{Var}(X) \ll a^{2} \cdot \log k$. We then show that $X \leq 2 \sqrt{2} \sqrt{k} a$.

Lemma 10: For any $k$ nodes $U$ placed in a square with side-length $a$, the length of $\operatorname{EMST}(U)$ is at most $2 \sqrt{2} \sqrt{k} a$.

Proof: Given $k$ nodes in the square, we will use Prim's algorithm to construct EMST: originally each node is a component, and then we iteratively find a shortest edge to connect two components to form a larger component until only one component is left. Consider the $(k+1-g)$-th step (for $1 \leq g \leq k$ ), which has $j$ connected components as input. For $g \geq 2$, if we partition the square into a $\lfloor\sqrt{g-1}\rfloor$ by $\lfloor\sqrt{g-1}\rfloor$ grid with side-length $\frac{a}{\lfloor\sqrt{g-1}\rfloor}$, then there is at least one cell that contains at least two connected components. This implies that the shortest edge connecting components at the $(k+1-g)$ th step is at most $\sqrt{2} \frac{a}{\lfloor\sqrt{g-1}\rfloor}$. Thus, the EMST has length at most

$$
\begin{aligned}
\sum_{j=2}^{k} \sqrt{2} \frac{a}{\lfloor\sqrt{g-1}\rfloor} & \leq \sum_{i=1}^{\sqrt{k-1}} \sqrt{2} \frac{a \cdot\left((i+1)^{2}-i^{2}-1\right)}{i} \\
& \leq 2 \sqrt{2} \sqrt{k-1} \cdot a
\end{aligned}
$$

This finishes the proof.

Bound the data copies: A straightforward lower-bound on the number of nodes (including leaf nodes) needed in a multicast tree spanning $k$ nodes randomly selected in a square of sidelength $a$ is $\tau \cdot \sqrt{k} \cdot \frac{a}{r}$ w.h.p. . This bound can be derived as follows: (1) the Euclidean length of a multicast tree is at least $\tau \cdot \sqrt{k} \cdot a$ w.h.p., and (2) the transmission range of each node is only $r$, thus, removing one tree edge incident on a leaf node will reduce the total edge length by at most $r$ and we will reduce the number of nodes by 1 . Thus, we have $C \geq$ $\tau \cdot \sqrt{k} \cdot a / r, w . h . p$. . Observe that similar analysis also shows that the number of nodes in a multicast tree $T$ spanning $k$ receivers is at most $4 \sqrt{2} \sqrt{k} a / r$. Although this bound on $C$ is much better than bound $C \geq k$ when $k=O\left(a^{2} / r^{2}\right)$, the bound can be further improved based on the following observation. When nodes on the multicast tree relay data from the source node to receivers, not only its downstream nodes of the multicast tree will receive the data, but also all its neighboring nodes (in communication graph $G$ ) will get a copy of the data. We will then analyze the number of nodes that will get the copy of the data. Given a multicast tree $T$, let $D(T)$ be the region covered by all transmitting disks of all transmitting nodes (internal nodes of $T$ ) in the multicast tree $T$. Observe that the leaf nodes do not contribute to $D(T)$ at all here. See Figure 1 (a) for illustration. Clearly, the area of $D(T)$, denoted by $|D(T)|$,

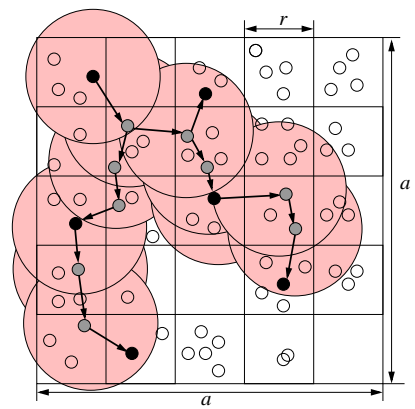

(a) Region $D(T)$

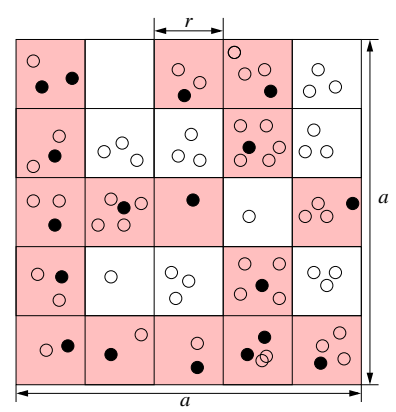

(b) Dense receivers
Fig. 1. (a) Region $D(T)$ covered by transmitting disks of internal nodes in multicast tree $T$. Here the solid black nodes are receivers of a source and gray nodes are Steiner nodes. (b) Partition of square with side-length $a$ into squarelets with side-length $r$. Here the solid black nodes are receivers of one source. Shaded squarelets are squarelets with at least one receiver.

is at most $|D(T)| \leq 2 r \cdot\|T\|+k \cdot \pi r^{2} / 2$ where $\|T\|$ is the 
total Euclidean length of all links in $T$. We will prove that the area of $D(T)$ is also at least $\frac{\tau \sqrt{k} a \cdot r}{c_{0}}, w . h . p$., for some constant $c_{0}$ independent of the network size.

Lemma 11: The area of the region $D(T)$, denoted by $|D(T)|$, w.h.p., is at least $\frac{\tau \sqrt{k} a \cdot r}{c_{0}}$ when the number of receivers per source $k<\theta_{1} \cdot \frac{a^{2}}{r^{2}}$. Here constant $d \leq 13, c_{0}=1 /(\rho \pi)$, $0<\rho<\frac{1}{6(d+1)}$, and

$$
\theta_{1}=\left(\frac{\tau(1-(6(d+1) \cdot \rho))}{6(d+1)+2}\right)^{2} .
$$

Proof: For any multicast tree $T$ spanning source node $v_{i}$ and the set of receivers $U_{i}$, for convenience, let $V(T)$ be the set of nodes in tree $T$; let $U_{i}^{\prime}=U_{i} \cup\left\{v_{i}\right\}$; let $I(T)$ be all the Steiner nodes used to connect them, i.e., $I(T)=$ $V(T) \backslash U_{i}^{\prime}$. Clearly the communication graph defined on $V(T)$ (where two nodes are connected iff their Euclidean distance is no more than $r$ ) is connected. We use $G_{T}$ to denote such induced graph. We will then build another multicast tree $T^{\prime}$ from $G_{T}$ to connect nodes $U_{i}^{\prime}$.

In graph $G_{T}$, we build a connected dominating set (CDS) using a method described in [2], [22]. Source node $s$ will be added to the CDS if it is not in the CDS. It has been proved in [2], [22] that, in the constructed CDS, each node on the CDS has a degree bounded by a constant, say $d$. For example, it can be shown that the degree of node in CDS is bounded by 13 if the method presented in [22] is used. The multicast tree $T^{\prime}$ is then a simple breadth-first-search tree computed from the CDS, rooted at the source node $s$.

We essentially will prove that each point from the region $D\left(T^{\prime}\right)$ is covered by at most a constant $c_{0}$ number of disks from the multicast tree $T^{\prime}$. For each point $p$ in the region, we divide the disk $D(p, r)$ centered at point $p$ with radius $r$ into 6 equal sized sectors. Thus, any pair of nodes falling into the same sector will be within distance of $r$ of each other, and thus connected in the original communication graph. Thus, for each point $p$, the number of disks from $D\left(T^{\prime}\right)$ that cover $p$ is at most $6(d+1)$. If it is at least $6(d+1)+1$, then at least one of the sectors will have at least $d+2$ nodes, which implies that any node in that sector will have degree at least $d+1$ in the induced CDS graph. This is a contradiction to the fact that the degree of induced CDS is bounded by $d$. Thus, the area of the region $D\left(T^{\prime}\right)$ is thus at least $\frac{\left|I\left(T^{\prime}\right)\right| \cdot \pi r^{2}}{6(d+1)}$, where $d$ is the degree bound on the induced CDS graph constructed. Here $\left|I\left(T^{\prime}\right)\right|$ is the number of internal nodes in tree $T^{\prime}$.

Notice that some leaf nodes in $T$ may become internal nodes in $T^{\prime}$; some internal nodes may not be used by tree $T^{\prime}$ at all. Let $A(T)$ be the region covered by all disks centered at all nodes of a tree $T$, including the leaf nodes. Let $\ell(T)$ be the number of leaf nodes in a tree $T$. Obviously, $|D(T)|+\ell(T)$. $\pi r^{2} \geq|A(T)| \geq\left|A\left(T^{\prime}\right)\right| \geq\left|D\left(T^{\prime}\right)\right|$. Thus,

$|D(T)| \geq\left|D\left(T^{\prime}\right)\right|-\ell(T) \cdot \pi r^{2} \geq \frac{\left|I\left(T^{\prime}\right)\right| \cdot \pi r^{2}}{6(d+1)}-\ell(T) \cdot \pi r^{2}$.

Obviously, $\ell(T) \leq k$. For a multicast tree $T^{\prime}$, there are at most $k$ leaf nodes. If we remove all edges in $T^{\prime}$ incident on leaf nodes, the total edge length of all edges left is at least $\left|T^{\prime}\right|-k \cdot r$. Thus, the number of internal nodes $\left|I\left(T^{\prime}\right)\right|$ in $T^{\prime}$ is at least $\frac{\left|T^{\prime}\right|-k \cdot r}{r}$. Notice that $T^{\prime}$ is a tree spanning the source node $s$ and all receivers $U_{i}^{\prime}$. Let $T^{\prime \prime}$ be the minimum length tree spanning $s=v_{i}$ and $k-1$ uniformly and randomly located points $P_{i}$. Then, w.h.p., $\left|T^{\prime \prime}\right| \geq \tau \sqrt{k} \cdot a$ [19]. Since adding all $k$ links $\left(v_{i, j}, p_{i, j}\right)$ to $T^{\prime}$ will result a spanning tree for $P_{i}$ and $v_{i}$, we have $\left|T^{\prime \prime}\right| \leq\left|T^{\prime}\right|+k \cdot r$. Thus, $\left|T^{\prime}\right| \geq \tau \sqrt{k} \cdot a-k r$. Thus, w.h.p., $\left|I\left(T^{\prime}\right)\right| \geq \frac{\left|T^{\prime}\right|}{r}-k \geq \frac{\tau \sqrt{k} \cdot a}{r}-2 k$. Thus,

$$
\begin{aligned}
|D(T)| & \geq \frac{\left|I\left(T^{\prime}\right)\right| \cdot \pi r^{2}}{6(d+1)}-\ell(T) \cdot \pi r^{2} \\
& \geq\left(\frac{\frac{\tau \sqrt{k} \cdot a}{r}-2 k}{6(d+1)}-k\right) \cdot \pi r^{2} \geq \rho \pi \tau \sqrt{k} \cdot a \cdot r
\end{aligned}
$$

when $k<\theta_{1} \cdot \frac{a^{2}}{r^{2}}$ and $\rho<\frac{1}{6(d+1)}$.

Based on Lemma 11, the expected number, denoted by $C$, of nodes from $V$ that is in the region $D(T)$ is at least

$$
\left(n-n^{\prime}\right)+|D(T)| \cdot \frac{n^{\prime}}{a^{2}} \geq\left(n-n^{\prime}\right)+\frac{\tau \cdot \sqrt{k} \cdot r \cdot n^{\prime}}{c_{0} a},
$$

where $n^{\prime}$ is the number of nodes not in tree $T$. Recall $n-n^{\prime}$ is the number of nodes in $T$, which is at most $4 \sqrt{2} \sqrt{k} a / r \leq 4 \sqrt{2 \theta_{1}} a^{2} / r^{2} \leq 4 \pi \sqrt{2 \theta_{1}} \frac{n}{\ln n}=O(n / \ln n)$ when $k \leq \theta_{1} a^{2} / r^{2}$. Here $a^{2} / r^{2} \leq n \pi / \ln n$ is from the necessary condition of having a connected random network. Thus, in the rest of paper, for simplicity, we always use $n$ to represent $n^{\prime}$, which will not affect the asymptotic behavior.

Lemma 12: Let $C_{j}$ be the number of nodes that get a "copy" of a given multicast bit. When $k \geq \frac{4 \pi c_{0}^{2}}{\tau^{2}}$,

$$
\operatorname{Pr}\left(C_{j}>\frac{\tau \cdot r \cdot \sqrt{k} \cdot n}{2 c_{0} a}\right)>1-\frac{1}{n^{2}} .
$$

Proof: Consider a multicast tree $T$. Notice that $n$ wireless nodes will be randomly distributed in a square region of sidelength $a$. Let $X_{i}=\{0,1\}$ be an indicator variable whether the $i$ th node $v_{i}$ will fall inside the region $D(T)$ for a multicast tree $T$. Clearly $\operatorname{Pr}\left(X_{i}=1\right)=\frac{|D(T)|}{a^{2}}$. Recall that, we already proved that, w.h.p., $|D(T)| \geq \frac{\tau \sqrt{k} a \cdot r}{c_{0}}$. Thus, $\operatorname{Pr}\left(X_{i}=1\right) \geq$ $\frac{\tau \sqrt{k} \cdot r}{c_{0} \cdot a}$. Obviously, $X=\sum_{i=1}^{n} X_{i}$ is the expected number of nodes falling inside the region $D(T)$, which is also the number $C$ of nodes that will get a copy of the data by multicast. Then the expected value $E(X) \geq \frac{\tau \sqrt{k} \cdot r \cdot n}{c_{0} \cdot a}$. Based on Lemma 2,

$$
\operatorname{Pr}\left(C \leq n \cdot \frac{|D(T)|}{2 a^{2}}\right) \leq e^{\frac{-n \cdot|D(T)|^{2}}{2 a^{4}}} \leq e^{-\frac{n \cdot \tau^{2} \cdot k \cdot r^{2}}{2\left(c_{0}\right)^{2} \cdot a^{2}}}
$$

Notice that to guarantee a connected network w.h.p., we have $a<\sqrt{\frac{\pi n}{\ln n}} \cdot r$ w.h.p.. Thus, when $n \rightarrow \infty$,

$$
\operatorname{Pr}\left(C \leq n \cdot \frac{|D(T)|}{2 a^{2}}\right) \leq e^{-\frac{n \cdot \tau^{2} \cdot k \cdot r^{2}}{2\left(c_{0}\right)^{2} \cdot a^{2}}} \leq e^{-\frac{\tau^{2} \cdot k \cdot \ln n}{2 \pi c_{0}^{2}}}=\frac{1}{n^{\frac{\tau^{2} \cdot k}{2 \pi c_{0}^{2}}}}
$$

Thus, when $\frac{\tau^{2} \cdot k}{2 \pi c_{0}^{2}} \geq 2$, i.e., $k \geq 4 \pi c_{0}^{2} / \tau^{2}$, we have

$$
\operatorname{Pr}\left(C>\frac{\tau \cdot r \cdot \sqrt{k} \cdot n}{2 c_{0} \cdot a}\right) \geq \operatorname{Pr}\left(C>n \cdot \frac{|D(T)|}{2 a^{2}}\right)>1-\frac{1}{n^{2}} .
$$

This finishes the proof. 
This implies that, with probability at least $1-\frac{1}{n}, C=$ $\max _{v_{i} \in \mathcal{S}} C_{i} \geq n \cdot \frac{|D(T)|}{2 a^{2}}$. Thus, we have

Theorem 13: The multicast capacity with $k-1$ receivers for $n$ nodes that are randomly and uniformly deployed in a square with side-length $a$ is at most $c_{1} \cdot \frac{a W}{r \sqrt{k}}$ for some constant $c_{1}$ when $k<\theta_{1} \cdot a^{2} / r^{2}$.

Proof: For $k<\frac{4 \pi c_{0}^{2}}{\tau^{2}}$, the capacity is bounded by the unicast capacity, which is $c_{1}^{\prime} \frac{a W}{r}$, implied by the results in [6]. Otherwise, the multicast capacity is at most $\frac{n \psi W}{C}$ and, w.h.p., $C \geq \frac{\tau \cdot r \cdot \sqrt{k} \cdot n}{2 c_{0} a}$ when $\frac{4 \pi c_{0}^{2}}{\tau^{2}} \leq k<\theta_{1} \cdot a^{2} / r^{2}$. Thus, the multicast capacity $\Lambda_{k}(n)$ is at most $\frac{n \psi W \cdot 2 c_{0} a}{\tau \cdot r \cdot \sqrt{k} \cdot n}$.

Generally the multicast capacity is at most $c_{1} \cdot \frac{a W}{r \sqrt{k}}$ for a constant $c_{1}=\max \left(\frac{2 c_{0} \psi}{\tau}, c_{1}^{\prime}\right)$.

Recall that we have proved that, to guarantee that we have a connected network w.h.p., we need $a \leq r \sqrt{\frac{n \pi}{\log n+\beta}}$ for $\beta \rightarrow$ $\infty$. Thus, letting $c_{2}=c_{1} \sqrt{\pi}$, we have the following theory:

Theorem 14: The multicast capacity for a random network of $n$ nodes, when $k<\theta_{1} \cdot a^{2} / r^{2}$, is at most

$$
\Lambda_{k}(n) \leq c_{2} \cdot \frac{\sqrt{n}}{\sqrt{\log n} \cdot \sqrt{k}} \cdot W=O\left(\frac{\sqrt{n}}{\sqrt{\log n} \cdot \sqrt{k}} \cdot W\right) .
$$

With $n_{s}$ multicast sessions, the per flow multicast capacity is at most

$\lambda_{k}(n)=\min \left\{W, \frac{\Lambda_{k}(n)}{n_{s}}\right\}=\min \left\{W, O\left(\frac{\sqrt{n}}{n_{s} \sqrt{\log n} \cdot \sqrt{k}} \cdot W\right)\right\}$.

Notice that Theorem 13 was proved under the assumption that $k \rightarrow \infty$. When this is not the case, we can prove that the per-flow multicast capacity $\vartheta$ (when each source node generates multicast data at rate $\vartheta$ ) also satisfies that $\lambda_{k}(n)=O\left(\frac{\sqrt{n}}{n_{s} \sqrt{\log n} \cdot \sqrt{k}} \cdot W\right)$ when $n_{s} \rightarrow \infty$. Since $k$ is constant in this case, we know that the per-flow multicast capacity is upper-bounded by the per-flow unicast capacity with $n_{s}$ unicast sessions. Thus, the per-flow multicast capacity is almost surely at most $O\left(\frac{\sqrt{n}}{n_{s} \sqrt{\log n}} \cdot W\right)$, which is same as $O\left(\frac{\sqrt{n}}{n_{s} \sqrt{\log n} \cdot \sqrt{k}} \cdot W\right)$ since $k$ is constant.

\section{When $k=\Omega\left(\frac{a^{2}}{r^{2}}\right)$}

In the previous subsection, we showed an upper bound of the multicast capacity when $k<\theta_{1} \cdot a^{2} / r^{2}$. In this subsection we will present an upper bound on multicast capacity when $k \geq \theta_{1} \cdot a^{2} / r^{2}$. We will essentially show that in this case, multicast is asymptotically equivalent to broadcast. Broadcast capacity of single-source of an arbitrary network has been studied in [9], [20]. In this paper, we will prove that the achievable integrated multicast capacity is only $\Theta(W)$ if an arbitrary $k$ subset of the $n$ nodes will serve as receivers for each possible source node $v_{i}$.

We partition the square of side-length $a$ into squarelets, each with side length $r$. The square will be partitioned into $M=$ $\left\lceil a^{2} / r^{2}\right\rceil$ squarelets, say $B_{1}, B_{2}, \cdots, B_{M}$. Recall that we will randomly select $k \geq \theta_{1} \cdot a^{2} / r^{2}$ receivers in the square region. See Figure 1 (b) for illustration.

Lemma 15: With probability at least $1-\frac{1}{\rho^{2} e^{\theta_{1} M}}$, at least $\rho \cdot M$ squarelets will have at least one receiver when $k \geq$ $\theta_{1} \cdot a^{2} / r^{2}$ for a constant $\theta_{1}$.
Proof: Let $X$ be the number of squarelets that do not have any receivers inside, and $A$ be a fixed fraction of squarelets, say $A=\rho \cdot M$ for a constant $0<\rho<1$. Define variable $X_{i}$

$$
X_{i}= \begin{cases}1 & \text { if squarelet } B_{i} \text { is empty of receivers, } \\ 0 & \text { if squarelet } B_{i} \text { is not empty of receivers. }\end{cases}
$$

Notice $X=\sum_{i=1}^{n} X_{i}$, and $\operatorname{Var}(X)=\operatorname{Var}\left(\sum_{i=1}^{M} X_{i}\right)=$ $\sum_{i=1}^{M} \sum_{j=1}^{M} \operatorname{Cov}\left(X_{i}, X_{j}\right)$, where $\operatorname{Cov}\left(X_{i}, X_{j}\right)=E\left(X_{i}\right.$. $\left.X_{j}\right)-E\left(X_{i}\right) E\left(X_{j}\right)$ is the covariance of variable $X_{i}$ and $X_{j}$. We then compute such $\operatorname{Cov}\left(X_{i}, X_{j}\right)$ for all possible pairs of $i$ and $j: \operatorname{Cov}\left(X_{i}, X_{i}\right)=E\left(X_{i}\right)-E\left(X_{i}\right)^{2}$ and $E\left(X_{i}\right)=\left(1-\frac{1}{M}\right)^{k}$; and $E\left(X_{i} \cdot X_{j}\right)=\left(1-\frac{2}{M}\right)^{k}$ if $i \neq j$. We can show that

$$
\operatorname{Var}(X) \leq M\left[\left(1-\frac{1}{M}\right)^{k}-\left(1-\frac{1}{M}\right)^{2 k}\right] .
$$

From Lemma 1, we have

$$
\operatorname{Pr}(X-E(X) \geq \rho \cdot M) \leq \frac{M\left[\left(1-\frac{1}{M}\right)^{k}-\left(1-\frac{1}{M}\right)^{2 k}\right]}{\rho^{2} M^{2}}
$$

Recall that $k \geq \theta_{1} \cdot M$. The expected value $E(X)$ of $X$, denoted by $\mu$, is $M \cdot\left(1-\frac{1}{M}\right)^{k} \leq M \cdot e^{-\theta_{1}}$. Thus,

$$
\operatorname{Pr}\left(X \geq\left(e^{-\theta_{1}}+\rho\right) \cdot M\right) \leq \frac{\left(\frac{1}{e}\right)^{\theta_{1}}-\left(\frac{1}{e}\right)^{2 \theta_{1}}}{\rho^{2}} \cdot \frac{1}{M}
$$

When $M \rightarrow \infty$, the probability goes to zero. We can also show that, w.h.p., there is at most a constant fraction of squarelets that will be empty of receivers.

Lemma 16: The union of the transmission disks of these $k$ nodes ( $k-1$ receivers and 1 source node) in a multicast will cover at least a constant fraction, say $0<\rho_{2} \leq 1$, of the deployment region.

Proof: Based on lemma 15, we know that among $M$ squarelets partitioned from the deployment region, there are at least $\rho \cdot M$ squarelets, each of which contains at least one receiver (or source) node inside. In each such a squarelet $B_{j}$, there is at least one receiver and thus at least one transmitting node in the multicast tree that covers this receiver. The transmitting node must lie inside this squarelet or 8 adjacent squarelets. On the other hand, each transmitting disk can cover receivers from at most 9 squarelets. Thus, we must have at least $\rho \cdot M / 9$ transmitting disks to cover receivers from $\rho \cdot M$ squarelets. Recall that the squarelet side-length is $r$, which implies that each point in the deployment region is covered by at most 9 such representative transmission disks. Thus, the total area covered by these representative transmission disks is at least $\rho \cdot M \cdot \pi r^{2} / 81$. Recall that the deployment region has area $a^{2}$ and $M=\left\lceil a^{2} / r^{2}\right\rceil$. Thus, the area of all transmission disks of all these $k$ nodes is at least $\rho_{2}=\frac{\rho \cdot \pi}{81}$ fraction of the total area of the deployment region.

Based on preceding lemmas, the following theorem is straightforward.

Theorem 17: When $k \geq \theta \cdot a^{2} / r^{2}$ for a constant $\theta$, the aggregated multicast capacity $\Lambda_{k}(n)$ w.h.p. is bounded from above by

$$
\Lambda_{k}(n) \leq \frac{W \cdot a^{2}}{\rho_{2} a^{2}}=\frac{W}{\rho_{2}}=O(W)
$$

where $\rho_{2}$ is a constant depending only on $\theta$. 
Notice that for broadcast, it has been proved in [9], [20] that the broadcast capacity is only $\Theta(W)$. Here we essentially prove that for multicast, when the number of receivers is large enough (at least $\Omega\left(\frac{a^{2}}{r^{2}}\right)$ ), the asymptotic multicast capacity is also only $O(W)$.

\section{LOWER BOUNDS ON MULTICAST CAPACITY With RANDOM NETWORKS}

In Section IV, we have derived upper bounds on the multicast capacity $\Lambda_{k}(n)$. In this section, we will derive asymptotically matching lower bounds on the multicast capacity $\Lambda_{k}(n)$. Specifically, we will provide a multicast scheme and prove that the multicast capacity achieved by our scheme matches the asymptotic upper bounds when the number of sessions $n_{\mathcal{S}}$ is large enough $\left(n_{\mathcal{S}}\right.$ could still be much smaller than $n$ ).

\section{A. Partition Square Using Squarelets}

Our multicast scheme is based on a good approximation of a minimum connected dominating set (MCDS) of a random network. First, partition the region into squarelets, each of sidelength $r / \sqrt{5}$. Thus, any two nodes from 2 adjacent squarelets (sharing a common side) will be able to communicate with each other directly. Randomly select one node from each squarelet. Clearly the set of selected nodes is a dominating set. If every squarelet has a node inside it, obviously, the set of selected nodes will form a CDS. The multicast will be routed on top of CDS. The CDS nodes can be scheduled in constant time-slots using TDMA. Notice, it is possible that, for some squarelet, there is no node inside, and thus, we cannot find a multicast tree $M T\left(U_{1}^{\prime}\right)$ later by Algorithm 1. We show that this almost surely cannot happen.

Lemma 18: There is a sequence of $\delta(n) \rightarrow 0$ such that

$$
\operatorname{Pr}(\text { Every squarelet contains a node }) \geq 1-\delta(n)
$$

Proof: Let $\mathcal{C}$ be the class of axis-aligned squares of sidelength $\frac{r}{\sqrt{5}}$. Notice that the probability that a node fall in such a square is $\frac{r^{2}}{5} \cdot \frac{1}{a^{2}}=\frac{r^{2}}{5 a^{2}}$. Recall that, to have a connected network, we almost surely have $r / a \geq \sqrt{\frac{\log n}{n \pi}}$. It is easy to show that the VC-dimension of $\mathcal{C}$ is at most 4 (it is at least $3)$. Hence, for all squarelets $S$,

$$
\begin{gathered}
\operatorname{Pr}\left(\sup _{S \in \mathcal{C}}\left|\frac{\# \text { of nodes in } S}{n}-\frac{r^{2}}{5 a^{2}}\right| \leq \epsilon(n)\right)>1-\delta(n), \text { if } \\
n \geq \max \left\{\frac{32}{\epsilon(n)} \cdot \log \frac{13}{\epsilon(n)}, \frac{4}{\epsilon(n)} \log \frac{2}{\delta(n)}\right\} .
\end{gathered}
$$

This condition (7) is satisfied when $\epsilon(n)=\frac{32 \log n}{n}, \delta(n)=\frac{2}{n}$.

$$
\operatorname{Pr}\left(\sup _{S \in \mathcal{C}}\left\{\# \text { of nodes in } S \geq \frac{n r^{2}}{5 a^{2}}-n \cdot \epsilon(n)\right\}\right)>1-\delta(n)
$$

Thus, if we choose $r$ and $a$ such that

$$
r \geq 14 \cdot a \cdot \sqrt{\frac{\log n}{n}}
$$

then $\frac{n r^{2}}{5 a^{2}}-n \cdot \epsilon(n) \geq 7 \log n$. Thus, we have

$$
\operatorname{Pr}(\forall \text { squarelet } S, \# \text { of nodes in } S \geq 7 \log n)>1-\frac{2}{n}
$$

The theorem then follows.

Notice that, generally, when $r=a \sqrt{\frac{\log n}{c \cdot n}}$, to make sure that every squarelet with side-length $r / \beta$ will have at least one node inside, it is sufficient to require that $c<\frac{1}{32 \beta^{2}}$.

For each node on the CDS, we show that every node can be scheduled to transmit once every $\Delta$ time-slots, where constant $\Delta$ depending only on $R$ and $r$. For each node $v$, consider a node $u$ whose transmission will interfere with the transmission of node $v$. Clearly node $u$ will be completely inside the disk centered at $v$ with radius $R+r$. Thus, the squarelet containing $u$ must be inside the disk centered at $v$ with radius $R+r+$ $\frac{\sqrt{2}}{\sqrt{5}} r<R+2 r$. Let $\Delta$ be the maximum number of nodes in CDS whose transmission will interfere with the transmission of a node $v$. Using the area argument, we have

$$
\Delta \leq \frac{\pi \cdot(R+2 r)^{2}}{r^{2} / 5}=5 \pi\left(2+\frac{R}{r}\right)^{2} .
$$

This property ensures that we can schedule the transmissions of all nodes in CDS by a TDMA manner such that all nodes will be able to transmit at least once in every $\Delta$ time slots. Notice that here $\Delta$ is a constant.

\section{B. When $k \leq \theta_{1} a^{2} / r^{2}$}

When the number of receivers, plus the source node, $k$ is at most $\theta_{1} \frac{a^{2}}{r^{2}}$, we will construct a multicast tree from CDS. Consider an instance of a random network $G=(V, E)$ and also an instance of multicast with $v_{1}$ as the source node and $U_{1}=\left\{v_{2}, v_{3}, \cdots v_{k}\right\}$ as the receiver nodes. Let $U_{1}^{\prime}=$ $\left\{v_{1}, v_{2}, v_{3}, \cdots v_{k}\right\}$. We will construct a multicast structure as following Algorithm 1.

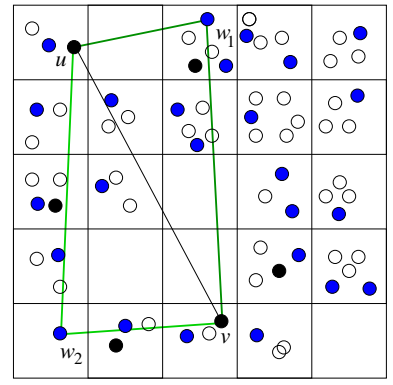

(a) Squarelets partition

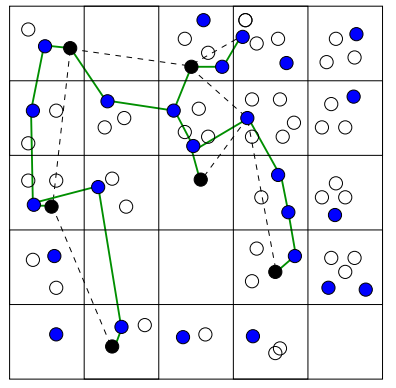

(b) Manhattan Routing Tree
Fig. 2. Partition of square with side-length $a$ into squarelets with side-length $r / \sqrt{5}$. For an edge $u v \in \operatorname{EMST}\left(U_{1}^{\prime}\right)$, find a node $w$ (either node $w_{1}$ or node $w_{2}$ which has same row as $u$ and same column as $v$ ) to connect them. Right figure illustrates a multicast tree constructed using Manhattan approach, where dotted lines denote an original spanning tree of nodes in a multicast session.

To show that Algorithm 1 achieves the asymptotically optimum multicast capacity, we need show that the total number of copies that a multicast bit is "received" by all nodes is at most $O\left(\frac{r \cdot \sqrt{k} \cdot n}{a}\right)$, which will be derived based on the upper bound on the area covered by all transmission disks in the multicast tree $M T\left(P_{i}\right)$. The following theorem will bound total Euclidean length of edges in the multicast tree $M T\left(P_{i}\right)$

Theorem 19: The total Euclidean length of the multicast tree $M T\left(P_{i}\right)$ is at most $c_{3} \sqrt{k} a$ for a constant $c_{3}$ depending only on $\theta_{1}$. 
$\overline{\text { Algorithm } 1 \text { Multicast Capacity Achieving Manhattan Rout- }}$ ing Based on a Squarelet for Nodes $U_{i}$

1: We partition the deployment square into squarelets, each with side length $r / \sqrt{5}$ (as in [15], [18], see Figure 1 (b) for illustration). Thus, we have $\left\lceil\frac{a}{r / \sqrt{5}}\right\rceil$ squarelets. Each squarelet is denoted by $(i, j)$ when it is the $i$ th column and $j$ th row.

2: Let $P_{i}=\left\{p_{i, 1}, p_{i, 2}, \cdots, p_{i, k}\right\}$ be the set of randomly and independently selected points used to find the terminals $U_{i}=\left\{v_{i, 1}, v_{i, 2}, \cdots, v_{i, k}\right\}$. Recall that $v_{i, j}$ is the closest node to point $p_{i, j}$.

3: We build an Euclidean spanning tree, denoted as $E S T(P)$, connecting points in $P_{i}$, using following method (also described in Lemma 10):

(1) Originally, $k$ nodes $P_{i}$ form $k$ components;

(2) repeat steps (3) and (4) for $g=1,2, \cdots, k-1$,

(3) for the $g$ th step, partition the deployment square into at most $k-g$ square-shaped-cells, each with side length $\left\lceil\frac{a}{\lfloor\sqrt{k-g}\rfloor}\right\rceil$

(4) find a cell that contains two points of $P_{i}$ that are from 2 different connected components and then connect them using Manhattan routing; merge these two connected components.

4: For each link $u v$ in the tree $\operatorname{EST}\left(P_{i}\right)$, assume that $u$ and $v$ are inside squarelet $\left(i_{u}, j_{u}\right)$ and squarelet $\left(i_{v}, j_{v}\right)$ respectively. Find a point $w$ in squarelet $\left(i_{v}, j_{u}\right)$ (or squarelet $\left.\left(i_{u}, j_{v}\right)\right)$, i.e., $u w v$ is a Manhattan path connecting $u$ and $v$. See Figure 2 (a) for illustration. The resulted structure by uniting all such paths for all links in $\operatorname{EST}\left(P_{i}\right)$ will serve the routing guideline for multicast.

5: For each edge $u w$ in $\operatorname{EST}\left(P_{i}\right)$, find a node in each of the squarelets that are crossed by line $u w$. We connect these nodes in sequence to form a path, denoted as $\mathbf{P}(u, v)$, connecting points $u$ and $v$. Notice that here such structure may not be a tree. If this is the case, we could remove the cycles that do not contain nodes from $P_{i}$. Denote the resulted tree as $M T\left(P_{i}\right)$.

6: For each receiver $v_{i, j}$, if it is not inside the squarelet $\mathbf{s}$ containing point $p_{i, j}$, let $v_{i, j}^{\prime}$ be the node selected inside the squarelet $\mathbf{s}$. Notice that, such $v_{i, j}^{\prime}$ exists for every squarelets, with probability at least $1-2 / n$. Node $v_{i, j}^{\prime}$ then relay the data to node $v_{i, j}$ (the relay takes at most 2 hops). The final tree (including these additional relays) are called multicast tree $M T R\left(U_{i}\right)$.

Proof: For $k$ nodes $P_{i}$ in a multicast session with source node $v_{i}$, we first constructed the Euclidean spanning tree $\operatorname{EST}\left(P_{i}\right)$. Same as the proof for Lemma 10, the total Euclidean length of all edges in $E S T\left(P_{i}\right)$ is at most $2 \sqrt{2} \sqrt{k} a$. For each edge $u v \in E S T\left(P_{i}\right)$, we will select a sequence of nodes (one in each of the squarelets crossed by segment $u w$ or $w v)$ to connect them. Assume that segment $u w$ crosses $x$ squarelets (including the one containing $u$ and $w$ ), and segment $w v$ crosses $y$ squarelets. Let $s$ be the size of the squarelet, which is $r / \sqrt{5}$ here. Then the path $\mathbf{P}(u, v)$ has Euclidean length at most $(x+y) s \cdot \sqrt{2}$ because the path has length at most $\sqrt{2} s$ in each crossed squarelet and it will cross $x+y$ squarelets. Additionally, we have

$$
\left\{\begin{array}{l}
(x \cdot s)^{2}+(y \cdot s)^{2} \geq\|u v\|^{2} \\
((x-1) \cdot s)^{2}+((y-1) \cdot s)^{2} \leq\|u v\|^{2}
\end{array}\right.
$$

Thus, $(x-1)+(y-1) \leq \sqrt{2} \sqrt{(x-1)^{2}+(y-1)^{2}}=$ $\sqrt{2} \frac{\|u v\|}{s}$. Then, $\mathbf{P}(u, v)$ has Euclidean length at most

$$
(x+y) s \cdot \sqrt{2} \leq 2\|u v\|+2 \sqrt{2} s .
$$

The total Euclidean length of all edges in $M T\left(P_{i}\right)$ is at most

$$
\begin{aligned}
& \sum_{u v \in E S T\left(P_{i}\right)} 2\|u v\|+2 \sqrt{2} s \\
= & 2\left\|\operatorname{EST}\left(P_{i}\right)\right\|+2 \sqrt{2} \frac{r}{\sqrt{5}} \cdot k \\
\leq & 2 \cdot(2 \sqrt{2} \sqrt{k} a)+\frac{2 \sqrt{10}}{5} \sqrt{k} \cdot a \cdot \frac{r}{a} \cdot \sqrt{k} \\
\leq & \left(4 \sqrt{2}+\frac{2 \sqrt{10}}{5} \cdot \sqrt{\theta_{1}}\right) \sqrt{k} \cdot a .
\end{aligned}
$$

The last inequality comes from $k \leq \theta_{1} a^{2} / r^{2}$. This finishes proof by setting $c_{3}=4 \sqrt{2}+\frac{2 \sqrt{10}}{5} \cdot \sqrt{\theta_{1}}$.

Given a squarelet, we define its flow-load as the total number of multicast sessions that will be routed through nodes inside this squarelet. We show that under our routing algorithm, for any squarelet, w.h.p., its flow-load is no more than $\Theta(\sqrt{k n \log n})$. To prove our claim, we first study a simple unicast case. Consider a grid of $L \times L$ squarelets. Consider a specific squarelet $\mathbf{s}$ that is of $i$ th row and $j$ th column in the squarelet-grid. Randomly pick two nodes $u$ and $v$ from the grid and connect them via Manhattan routing. Let $X_{\mathbf{s}}$ be a random variable denoting whether the Manhattan routing will use nodes from the squarelet $\mathbf{s}$. Let $p_{\mathbf{s}}(L)$ denote the probability that the Manhattan routing will use nodes from the squarelet s, i.e., $p_{\mathbf{s}}(L)=\operatorname{Pr}\left(X_{\mathbf{s}}=1\right)$. Then

$$
p_{\mathbf{s}}(L)=\frac{i-1}{L^{2}} \cdot \frac{L-i+1}{L}+\frac{j-1}{L^{2}} \cdot \frac{L-j+1}{L} .
$$

Here $\frac{i-1}{L^{2}} \cdot \frac{L-i+1}{L}$ (resp. $\frac{j-1}{L^{2}} \cdot \frac{L-j+1}{L}$ ) is the probability that squarelet $\mathbf{s}$ is used when $u$ (resp. $v$ )is on the same row (resp. column) as $\mathbf{s}$. It is easy to show that

$$
\frac{2}{L^{2}} \leq p_{\mathbf{s}}(L) \leq \frac{2}{L} \text {. }
$$

Let us now study the number of times that a specific squarelet $\mathbf{s}$ is used by our routing structure for multicast.

Lemma 20: Given a squarelet $\mathbf{s}$, the probability that a random multicast flow will be routed via the squarelet $\mathbf{s}$ is at most $c_{6} \sqrt{k} \cdot \frac{r}{a}$.

Proof: Recall that we will construct the Euclidean spanning tree as the method described in Algorithm 1 and then find multicast routing structure as Algorithm 1. For a given multicast session, this squarelet $\mathbf{s}$ may be used in any one of the $k$ steps to build the spanning tree. For step $g$ (with $1 \leq g \leq k-1$ ), recall that we will partition the square with side-length $a$ into $\lfloor\sqrt{k-g}\rfloor^{2} \leq k-g$ cells, each with side-length $\frac{a}{\lfloor\sqrt{k-g}\rfloor}$. From pigeonhole principle, there exists a cell that contains two nodes, say $u$ and $v$, from two different 
connected components. We will connect them and merge these two connected components. Here we will connect $u$ and $v$ using Manhattan routing as illustrated in Figure 2. Let $X_{\mathbf{s}, g}$ be the indicator whether the specific squarelet $\mathbf{s}$ is used in this $g$ th step. Clearly, the probability that $\operatorname{Pr}\left(X_{\mathbf{s}, g}=1\right)$ is

$$
\operatorname{Pr}\left(X_{\mathbf{s}, g}=1\right)=\frac{1}{\lfloor\sqrt{k-g}\rfloor^{2}} \cdot p_{\mathbf{s}}\left(\left\lceil\frac{\frac{a}{\lfloor\sqrt{k-g}\rfloor}}{r / \sqrt{5}}\right\rceil\right),
$$

where $\frac{1}{\lfloor\sqrt{k-g}\rfloor^{2}}$ is the probability that the cell containing squarelet $\mathbf{s}$ is used, and $p_{\mathbf{s}}\left(\left\lceil\frac{\frac{a}{\lfloor\sqrt{k-g}\rfloor}}{r / \sqrt{5}}\right\rceil\right)$ is the probability that squarelet $\mathbf{s}$ is used when that cell containing $\mathbf{s}$ is used. Here $\left\lceil\frac{\frac{a}{\lfloor\sqrt{k-g}\rfloor}}{r / \sqrt{5}}\right\rceil$ is the number of squarelets per row in a cell, i.e., the value of $L$ in formula 10 . Thus,

$$
\begin{aligned}
p & =\operatorname{Pr}\left(X_{\mathbf{s}}=1\right) \leq \sum_{g=1}^{k-1} \operatorname{Pr}\left(X_{\mathbf{s}, g}=1\right) \\
& =\sum_{g=1}^{k-1} \frac{1}{\lfloor\sqrt{k-g}\rfloor^{2}} \cdot p_{\mathbf{s}}\left(\left\lceil\frac{\frac{a}{\lfloor\sqrt{k-g}\rfloor}}{r / \sqrt{5}}\right\rceil\right) \leq \frac{4 \sqrt{10}}{5} \sqrt{k} \cdot \frac{r}{a}
\end{aligned}
$$

The theorem follows by setting $c_{6}=\frac{4 \sqrt{10}}{5}$.

Recall that, the multicast rooted at $v_{i}$ will first randomly and independently select $k-1$ points $P_{i}^{\prime}$. To use the VC Theorem, we will construct a multicast tree using the union of node $v_{i}$ and $P_{i}^{\prime}$ as $P_{i}$, which is the input of Algorithm 1. The data will then be relayed to every node $v_{i, j}$ if it did not receive the data before. Thus, the points used to construct the multicast trees $M T\left(P_{i}\right)$ for different source nodes are independently and randomly chosen in the deployment region. Notice that, given $k$ terminals $U$, the multicast tree $M T(U)$ constructed by Algorithm 1 can be uniquely defined by its terminals $U$, thus has dimension $2 k$. In other words, every point in $2 k$-dimensional cube (with side-length $a$ ), corresponds to a multicast tree. Given any 2-dimensional axis-aligned square $h$ (not necessarily the squarelet produced by partitioning the deployment region), let set $T(h)$ be the set of multicast trees (equivalently, the set of points in $R^{2 k}$ defining these trees) that will intersect the square $h$ (i.e., one of its edges will have point inside $h$ ). Let

$$
\mathcal{F}=\left\{T(h) \mid h \text { is an axis-aligned square with size } \frac{r}{\sqrt{5}}\right\} .
$$

In the appendix, we show that the VC-dimension of $\mathcal{F}$ is

$$
d=\Theta(\log k)
$$

Theorem 21: Assume that there are $N$ random multicast sessions with $N$ satisfying inequality (15). There is a sequence of $\delta(n) \rightarrow 0$ such that

$$
\begin{aligned}
& \operatorname{Pr}\left(\forall \text { squarelet } \mathbf{s}, \# \text { of flows using } \mathbf{s} \leq \frac{3 \sqrt{c_{6}} N}{2} \sqrt{k} \frac{r}{a}\right) \\
\geq & 1-\delta(n)
\end{aligned}
$$

Proof: The terminals used to construct multicast trees are i.i.d. variables. Then the multicast trees are i.i.d. variables. Thus, we can use the VC-Theorem. Recall that, given a square $h$, the probability that a multicast tree will cross $h$ is at most $c_{6} \sqrt{k} \cdot \frac{r}{a}$ (see Lemma 20). Hence, for all squarelets $S$,

$$
\begin{gathered}
\operatorname{Pr}\left(\sup _{S \in \mathcal{F}}\left|\frac{\# \text { of flows using } S}{N}-P(S)\right| \leq \epsilon(n)\right)>1-\delta(n) \\
\quad \text { when } N \geq \max \left\{\frac{8 d}{\epsilon(n)} \cdot \log \frac{13}{\epsilon(n)}, \frac{4}{\epsilon(n)} \log \frac{2}{\delta(n)}\right\} .
\end{gathered}
$$

Here $d$ is the VC-dimension of $\mathcal{F}$ and $P(S)$ is the probability of a set $S$, which is at most $c_{6} \sqrt{k} \cdot \frac{r}{a}$. Thus,

$$
\operatorname{Pr}\left(\sup _{S \in \mathcal{F}} \frac{\# \text { of flows using } S}{N} \leq \frac{c_{6} \sqrt{k} r}{a}+\epsilon(n)\right)>1-\delta(n)
$$

whenever condition (13) is satisfied. Let

$$
\epsilon(n)=\frac{c_{6} \sqrt{k} r}{2 a}, \text { and } \delta(n)=\frac{2}{n} .
$$

Let $a / r=\sqrt{c \cdot n / \log n}$ for $c \in(0,1 / 160)$. For condition (13) to be true, it is sufficient that $N \geq \max \left\{\frac{8 d \sqrt{c}}{c_{6}} \sqrt{\frac{n}{k \log n}}\right.$. $\left.\log \left(\frac{26 \sqrt{c}}{c_{6}} \sqrt{\frac{n}{k \log n}}\right), \frac{8 \sqrt{c}}{c_{6}} \sqrt{\frac{n}{k \log n}} \log n\right\}$. When $n$ is sufficiently large, it is sufficient that

$$
N \geq \frac{4 d \sqrt{c}}{c_{6}} \sqrt{\frac{n \log n}{k}} .
$$

This finishes the proof.

Notice that condition (15) can always be satisfied as long as we have $n_{s}=\Omega\left(\sqrt{\frac{n \log n}{k}} \cdot \log k\right)$ multicast sessions. Thus, if we assign data rate

$$
\vartheta=\frac{W}{2 \Delta \frac{3 \sqrt{c_{6}} N}{2} \sqrt{k} \frac{r}{a}}=O\left(\frac{W}{\log n}\right)
$$

to each of the $N$ multicast sessions (with random source node and random terminal points), with probability at least $1-\frac{2}{n}$, the total data rate that will be routed through every squarelet is at most $\frac{W}{2 \Delta}$. Recall that, for a multicast rooted at $v_{i}$, our routing will first send data to squarelets containing points $P_{i}$. Then for $1 \leq j \leq k-1$, we need forward the data from the squarelet containing the point $p_{i, j}$ to the nearest node $v_{i, j}$ respectively. Notice that we have proved that, for every squarelet, with probability at least $1-\frac{2}{n}$, there are at least $\Theta(\log n)$ nodes inside. Thus, with probability at least $1-\frac{2}{n}$, every data can be transferred to some node inside the squarelet. Thus, counting this last-hop relay, with probability at least $1-\frac{2}{n}$, the total data rate every squarelet has to route is at most $2 \frac{W}{2 \Delta}=\frac{W}{\Delta}$. Thus, these flows can be supported by a TDMA scheduling.

Theorem 22: Assume $k \leq \theta_{1} \frac{a^{2}}{r^{2}}$, there are $n_{s}$ random multicast sessions and $n_{s} \geq \frac{4 d \sqrt{c}}{c_{6}} \sqrt{\frac{n \log n}{k}}$. With probability at least $\left(1-\frac{2}{n}\right)^{2} \geq 1-\frac{4}{n}$, the achievable aggregated multicast capacity is at least

$$
\Lambda_{k}(n)=\frac{W}{3 \sqrt{c_{6}} \Delta \sqrt{k} \frac{r}{a}}=\Theta\left(\frac{W}{\sqrt{k}} \cdot \frac{a}{r}\right) .
$$

Theorem 23: Assume $k \leq \theta_{1} \frac{a^{2}}{r^{2}}$, there are $n_{s}$ random multicast sessions and $n_{s} \geq \frac{4 d \sqrt{c}}{c_{6}} \sqrt{\frac{n \log n}{k}}$. With probability 
at least $\left(1-\frac{2}{n}\right)^{2} \geq 1-\frac{4}{n}$, the achievable per-flow multicast capacity is at least

$$
\lambda_{k}(n)=\frac{W}{3 \sqrt{c_{6}} \Delta} \cdot \frac{a}{n_{s} r \sqrt{k}}=\Theta\left(\frac{W}{n_{s} \sqrt{k}} \cdot \frac{a}{r}\right) .
$$

By setting $\frac{a}{r}=\sqrt{\frac{c \cdot n}{\log n}}$ for a constant $c \leq 1 / 160$, we know that the achievable aggregated multicast capacity is at least $\Theta\left(\sqrt{\frac{n}{k \log n}} \cdot W\right)$, and the per-flow multicast capacity is at least $\Theta\left(\sqrt{\frac{n}{k \log n}} \cdot \frac{W}{n_{s}}\right)$ (which is smaller than $O\left(\frac{W}{\log n \cdot \log k}\right)$, thus feasible) when there are at least $n_{s}=\Omega\left(\sqrt{\frac{n \log n}{k}} \cdot \log k\right)$ randomly and independently chosen flows. Thus, we have

Corollary 24: The multicast capacity for a random network of $n$ nodes, when $k<\theta_{1} \cdot a^{2} / r^{2}$, is at least

$$
\Lambda_{k}(n) \geq c_{2}^{\prime} \cdot \frac{\sqrt{n}}{\sqrt{\log n} \cdot \sqrt{k}} \cdot W=\Omega\left(\frac{\sqrt{n}}{\sqrt{\log n} \cdot \sqrt{k}} \cdot W\right),
$$

with $c_{2}^{\prime}=\frac{\sqrt{c}}{3 \Delta \sqrt{c_{6}}}$. The per-flow multicast capacity is at least

$$
\lambda_{k}(n)=\frac{\Lambda_{k}(n)}{n_{s}}=\Omega\left(\frac{\sqrt{n}}{n_{s} \sqrt{k \log n}} \cdot W\right),
$$

with $n_{s}=\Omega\left(\sqrt{\frac{n \log n}{k}} \cdot \log k\right)$ random multicast sessions.

It remains an interesting question whether we can lower the requirement for $n_{s}$ to guarantee the correctness of this corollary.

\section{When $k \geq \theta_{1} \frac{a^{2}}{r^{2}}$}

In this case, we have proved that the upper bound on the total multicast capacity is only $W / \varrho_{2}=\Theta(W)$. Obviously, the total multicast capacity is at least the lower bound of the capacity for broadcast. In [9], they present a broadcast scheme to achieve capacity $\Theta(W)$. Thus, we have

Theorem 25: The total multicast capacity $\Lambda_{k}(n)$ achievable by all multicast flows is at least $c_{7} W$ when $k=\Omega\left(a^{2} / r^{2}\right)$, where $c_{7}=\frac{1}{\Delta}$ and constant $\Delta$ is the maximum number of CDS nodes that are within interference range $R$ of a node.

\section{Other Multicasts}

\section{A. Capacity Bound for Group Multicast}

In previous sections we have studied the asymptotic multicast capacity by assuming that we randomly select $k-1$ receivers for each multicast session. In this section, we study the multicast capacity of so-called $k$-group multicast: for each source node $v_{i}$, there are $k-1$ groups of receivers $g_{i, 1}, g_{i, 2}$, $\cdots, g_{i, k-1}$. The receivers in each group $g_{i, j}$ are covered by a disk with radius $\delta \cdot r$ for a constant $\delta$ and centered at one of the receivers in the group. We assume that the center node in each group is randomly selected. The number of nodes in each group could be arbitrary. For simplicity, let node $z_{i, j}$ be the center node of group $g_{i, j}$. We then study the multicast capacity for group-multicast when each node $v_{i}$ will have $k-1$ randomly selected groups and it wants to send data with rate $\vartheta_{i}$ to all receivers in these $k-1$ groups.

As the case when each group has only one node, when $k \geq \theta_{1} a^{2} / r^{2}$, it is easy to prove that the capacity for groupmulticast is at most $W / \varrho_{2}$ as Theorem 17. Clearly, a simple broadcast based on the connecting dominating set constructed previously will also achieve a capacity for group-multicast at least $\frac{W}{\Delta}$. Thus, we have

Theorem 26: For group-multicast, when $k \geq \theta_{1} a^{2} / r^{2}$ for any constant $\theta_{1}>0$, the capacity of group-multicast is at most $W / \varrho_{2}$ and at least $\frac{W}{\Delta}$.

First, for group-multicast, a multicast tree has to reach the center node $z_{i, j}$ of each group $g_{i, j}$. Then from Theorem 13, the capacity of group-multicast is at most $c_{1} \cdot \frac{a W}{r \sqrt{k}}$, w.h.p., when $k<\theta_{1} \cdot a^{2} / r^{2}$. We then show how to design multicast routing for the group-multicast problem: we first apply our multicast scheme for traditional multicast when nodes $z_{i, j}, 1 \leq j \leq k-1$, are receivers for source node $v_{i}$. We then let node $z_{i, j}$ multicast locally to all receivers in the group $g_{i, j}$. We already proved in Theorem 19, the total length of the multicast tree to span these randomly selected nodes $z_{i, j}$ is at most $c_{3}\left\|E M S T\left(U_{i}^{\prime}\right)\right\|$ w.h.p. . Recall that $\left\|E M S T\left(U_{i}^{\prime}\right)\right\| \leq \frac{3 \tau(2) \sqrt{k} \cdot a}{2}$ w.h.p. . Notice that the total area covered by transmitting disks of relay nodes used for relaying data from each $z_{i, j}$ to receivers in its group is at most $\pi(\delta+1) r^{2}$. Then, the area covered by all transmitting disks for a multicast session is at most

$$
\begin{aligned}
& 2 r \cdot\left|M T\left(P_{i}\right)\right|+(k-1) \cdot \pi(\delta+1) r^{2} \\
\leq & \left.2 r \cdot c_{3} \frac{3 \tau(2) \sqrt{k} \cdot a}{2}+k \cdot \pi(\delta+1) r^{2} \quad \text { (w.h.p. }\right) \\
\leq & \left(\theta_{1} \pi(\delta+1)^{2}+3 c_{3} \tau(2)\right) \cdot \sqrt{k} \cdot a \cdot r
\end{aligned}
$$

The last inequality comes from the fact that $k \leq \theta_{1} \frac{a^{2}}{r^{2}}$. For convenience, let $c_{8}=\theta_{1} \pi(\delta+1)^{2}+3 c_{3} \tau(2)$. Then using Lemma 2, we can prove that the number of nodes that will get a copy of the data from one multicast session is at most $c_{8} \sqrt{k} \cdot a \cdot r \cdot \frac{2 n}{a^{2}}=2 c_{8} \cdot n \cdot \sqrt{k} \cdot \frac{r}{a}$. Thus, we have

Theorem 27: When $k \leq \theta_{1} a^{2} / r^{2}$, the aggregated multicast capacity for group-multicast with $k-1$ groups is at most $c_{1}$. $\frac{a W}{r \sqrt{k}}$, and is at least $c_{9} \frac{a \cdot W}{r \sqrt{k}}$, w.h.p.. Here constant $c_{9}=2 c_{8} \Delta$.

\section{B. Capacity Bounds for d-dimensional Networks}

It is not difficult to extend our capacity bounds to networks in $d$ dimensions. Assume that the network nodes are randomly deployed in a $d$-dimensional cube with side-length $a$ and every node has a transmission range $r$ and an interference range $R$. The total Euclidean length of edges in any tree spanning $k$ nodes randomly distributed in the $d$-dimensional cube is, w.h.p., at least $\Omega\left(k^{\frac{d-1}{d}} a\right)$ [19]. Similar to Lemma 10, we can show that the total Euclidean length of the edges in EMST spanning any $k$ nodes is at most $O\left(k^{\frac{d-1}{d}} a\right)$. The total volume of the region shaded by all transmitting spheres of all internal nodes of a multicast tree is then at the order of $\Theta\left(k^{\frac{d-1}{d}} \cdot \frac{a}{r} \cdot r^{d}\right)$. Thus, the number of nodes that will get a copy of the multicast data is at the order of $\Theta\left(k^{\frac{d-1}{d}} \cdot \frac{a}{r} \cdot r^{d} \cdot \frac{n}{a^{d}}\right)$, which is at most $n$ when $k \leq \theta_{d}\left(\frac{a}{r}\right)^{d}$ for some constant $\theta_{d}$ depending only on dimension $d$. This implies that the aggregated multicast capacity of $n_{s}$ multicast sessions is at the order of $\Theta\left(\frac{n W}{n k \frac{d-1}{d}\left(\frac{r}{a}\right)^{d-1}}\right)=\Theta\left(\frac{W}{k^{\frac{d-1}{d}}} \cdot\left(\frac{a}{r}\right)^{d-1}\right)$. To show the lower-bound on achievable multicast capacity, we will also partition the cube into cubelets with side length $r / \sqrt{3+d}$ 
such that two nodes inside two cubelets sharing a $(d-1)$ dimensional facet can communicate with each other directly. We can then show that, given a specific cubelet, the probability that a random multicast flow will be routed through this cubelet is $k^{\frac{d-1}{d}} \cdot\left(\frac{r}{a}\right)^{d-1}$. Thus, we have the following theorem:

Theorem 28: Assume that $n$ nodes are randomly placed in a $d$-dimensional cube of side-length $a$ and each node has a fixed transmission range $r$. The aggregated multicast capacity of $n_{s}$ random multicast sessions (each with $k$ terminals) is

$$
\Lambda_{k}(n)= \begin{cases}\Theta\left(\left(\frac{a}{r}\right)^{d-1} \cdot \frac{W}{k^{\frac{d-1}{d}}}\right) & \text { when } k \leq \theta_{d}\left(\frac{a}{r}\right)^{d}, \text { and } \\ \Theta(W) & n_{s}=\Omega\left(\left(\frac{a}{r}\right)^{d-1} \frac{\log n}{k^{\frac{d-1}{d}}}\right) . \\ & \text { when } k \geq \theta_{d}\left(\frac{a}{r}\right)^{d}\end{cases}
$$

In the above formula, $\theta_{d}$ is a constant depending on dimension $d$.

Recall that Penrose [16] showed that the asymptotic length of the longest edge of EMST of $n$ nodes randomly placed in a $d$-dimensional cube of unit side-length is $\left(\frac{2(1-1 / d) \log n}{\theta n}\right)^{1 / d}$ where $\theta$ is the volume of the unit ball in $d$-dimension. Thus, to get a connected network, we need $a$ and $r$ satisfy the following condition, w.h.p.,

$$
\frac{a}{r} \leq c_{d} \sqrt[d]{\frac{n}{\log n}}
$$

for some constant $c_{d}$ depending on dimension $d$. Thus, we have the following theorem.

Theorem 29: If we can choose the transmission range $r$ or the deployment region $a$, the aggregated multicast capacity of $n_{s}$ multicast sessions for $n$ randomly placed nodes in a $d$-dimensional cube of side-length $a$ is

$\Lambda_{k}(n)= \begin{cases}\Theta\left(W \cdot\left(\frac{n}{k \log n}\right)^{1-\frac{1}{d}}\right) & \text { when } k \leq \theta_{d}\left(c_{d}\right)^{d} \frac{n}{\log n}, \text { and } \\ \Theta(W) & n_{s}=\Omega\left(\left(\frac{n}{k \log n}\right)^{1-\frac{1}{d}} \cdot \log n\right) . \\ & \text { when } k \geq \theta_{d}\left(c_{d}\right)^{d} \frac{n}{\log n}\end{cases}$

\section{LITERATURE REVIEWS}

For a given statistical description of the network, a set of constraints (such as power per node, link capacity, etc.), and a list of desired communication pairs, the capacity region is the closure of all rate tuples that can be achieved simultaneously. Here a rate tuple specifies the rate for each of the desired communications. Kyasanur and Vaidya [13] studied the capacity region on random multi-hop multi-radio multi-channel wireless networks. On the other aspect, several papers [1], [11] recently studied how to satisfy a certain traffic demand vector from all wireless nodes by a joint routing, link scheduling, and channel assignment under certain wireless interference models.

Gupta and Kumar [6] studied the asymptotic unicast capacity of a multi-hop wireless networks for two different models. When each wireless node is capable of transmitting at $W$ bits per second using a constant transmission range, the throughput obtainable by each node for a randomly chosen destination is $\Theta\left(\frac{W}{\sqrt{n \log n}}\right)$ bits per second under a non-interference protocol, where $n$ in number of nodes. If nodes are optimally assigned and transmission range is optimally chosen, even under optimal circumstances, the throughput is only $\Theta\left(\frac{W}{\sqrt{n}}\right)$ bits per second for each node. Similar results also hold for physical interference model. Kulkarni and Viswanath [12] obtained a stronger (almost sure) version of the $\sqrt{n \log n}$ throughput for random node locations in a fixed area obtained by Gupta and Kumar. For Gaussian channel model, Franceschetti et al. [4] shows that a rate $1 / \sqrt{n}$ is achievable in networks of randomly located nodes (not only some arbitrarily placed nodes).

Grossglauser and Tse [5] recently showed that mobility actually can help to improve the unicast capacity if we allow arbitrary large delay. Their main result shows that the average long-term throughput per source-destination pair can be kept constant even as the number of nodes per unit area increases. Notice that this is in sharp contrast to the fixed network scenario (when nodes are static after random deployment). Essentially, the result presented in [5] still obey the capacity bound proposed in [6]: the capacity is improved because the average distance $\bar{L}$ a packet has to be transmitted is reduced from $\Theta(1)$ in [6] to $\Theta(r(n))$ in [5]. In summary, for random networks, under the protocol model, the achievable per-flow throughput capacity $\lambda(n)$ and the average travel distance $\bar{L}$ satisfies $\lambda(n) \cdot \bar{L} \leq \Theta\left(\frac{W}{\Delta^{2} n \cdot r(n)}\right)$. Similar phenomenon has also been observed in [14].

Broadcast capacity of an arbitrary network has been studied in [9], [20]. They essentially show that the broadcast capacity of a given network is $\Theta(W)$ for single source broadcast and the achievable broadcast capacity per flow is only $\Theta(W / n)$ if each of the $n$ nodes will serve as source node. The capacity $\Theta(W)$ is achieved by constructing a CDS in which we can schedule every node in CDS to transmit at least once in constant time slots. This capacity bounds also apply to random networks. Keshavarz-Haddad et al. [10] studied the broadcast capacity with dynamic power adjustment for physical interference model.

Multicast capacity was not fully studied in the literature. Jacquet and Rodolakis [8] studied the scaling properties of multicast for random wireless networks. They essentially studied the normalized multicast cost, which is defined as the ratio of the number of links in the multicast tree over the average route length from a random source in the multicast group to a random destination in the multicast group. They briefly showed that the maximum rate at which a node can transmit multicast data is $O\left(\frac{W}{\sqrt{k n \log n}}\right)$. At the same time as [15], Shakkottai et al. [18] studied the multicast capacity of random networks when the number of multicast sources is $n^{\epsilon}$ for some $\epsilon>0$, and the number of receivers per multicast flow is $n^{1-\epsilon}$. They assume the protocol interference model and use the dense random network model. They show that the sum of the source rates $\Lambda(n)$ that the network can support is $O\left(\frac{\sqrt{n^{\epsilon}}}{\sqrt{\log n}}\right)$ w.h.p., with a per flow throughput capacity of $O\left(\frac{1}{\sqrt{n^{\epsilon} \log n}}\right)$ w.h.p.. Notice that this result can be implied by our results using $n_{s}=n^{\epsilon}$ and $k=n^{1-\epsilon}$. To achieve the upper bound, they propose a simple and novel routing architecture, called the multicast comb, to transfer multicast data in the network. 


\section{CONCLUSIONS}

In this paper, we derived analytical upper bounds and lower bounds on multicast capacity of a wireless network when all nodes are uniformly and randomly deployed in a square region with side-length $a$, and all nodes have the same transmission range $r$. Under protocol interference model, we showed that the total multicast capacity is only $\Theta\left(\sqrt{\frac{n}{\log n}} \cdot \frac{W}{\sqrt{k}}\right)$ when $k=O\left(\frac{n}{\log n}\right)$; and is $\Theta(W)$ when $k=\Omega\left(\frac{n}{\log n}\right)$. We also studied the multicast capacity for group-multicast and for $d$ dimensional networks. It is not difficult to show that all our results still apply when the deployment region is a square with side length $a=1$, while the transmission range is selected appropriately, i.e., $r=\Theta\left(\sqrt{\frac{\log n}{\pi n}}\right)$. It is also not difficult to show that our results still hold when $r=1$ while the deployment region has a bounded aspect ratio such as a disk. We can also show that our results still hold when physical interference model is used. The basic idea is to show that the simultaneous transmitting nodes will be separated by a certain distance $R_{0}$ for any interference-free scheduling and, when the distance between transmitting nodes is at least $R_{1}$ and the link length is at most $r$, then no interference will occur.

There are several interesting questions left for study. The first question is what is the multicast capacity when the link capacity is not uniform: shorter links will have larger capacity, such as Gaussian channel capacity. We conjecture that per-flow multicast capacity will be $\Theta\left(\frac{1}{\sqrt{k n}}\right)$ when Gaussian channel rate is used and $k=O\left(\frac{n}{\log ^{b}(n)}\right)$ for some integer $b>1$. The second question is what is the multicast capacity when the receivers of any source $v$ are inside $D(v, B)$ for a bound $B$. The third question is what is the multicast capacity bound when the network is not necessarily connected. Last but not the least question is to study the tradeoffs between the delay and multicast capacity for random mobile networks.

\section{ACKNOWLEDGMent}

I am deeply grateful to YaJun Wang and WangSen Feng for initial discussing on the VC-dimension of multicast trees, to ShaoJie Tang and Ophir Frieder for some discussions on capacity in general, and to anonymous reviewers for their constructive comments for improving the paper.

\section{REFERENCES}

[1] M. Alicherry, R. Bhatia, and L. E. Li. Joint channel assignment and routing for throughput optimization in multi-radio wireless mesh networks. In ACM MobiCom '05, pages 58-72.

[2] K. Alzoubi, X.-Y. Li, Y. Wang, P.-J. Wan, and O. Frieder. Geometric spanners for wireless ad hoc networks. IEEE Transactions on Parallel and Distributed Processing, 14(4):408-421, 2003.

[3] D.-Z. Du and F.-K. Hwang. A proof of the gilbert-pollak conjecture on the steiner ratio. Algorithmica, 7((2,3)):121-135, 1992.

[4] M. Franceschetti, O. Dousse, D. N.C.Tse, and P. Thiran. Closing the gap in the capacity of wireless networks via percolation theory, 53(3), 2007.

[5] M. Grossglauser and D. Tse. Mobility increases the capacity of ad hoc wireless networks. IEEE/ACM Transactions on Networking (TON), 10(4):477-486, 2002.

[6] P. Gupta and P. Kumar. Capacity of wireless networks. IEEE Transactions on Information Theory, IT-46:388-404, 1999.

[7] P. Gupta and P. R. Kumar. Critical power for asymptotic connectivity in wireless networks. Stochastic Analysis, Control, Optimization and Applications: A Volume in Honor of W.H. Fleming, W. M. McEneaney, G. Yin, and Q. Zhang (Eds.), 1998.
[8] P. Jacquet and G. Rodolakis. Multicast scaling properties in massively dense ad hoc networks. In ICPADS '05: Proceedings of the 11th International Conference on Parallel and Distributed Systems - Workshops (ICPADS'05), pages 93-99, 2005.

[9] A. Keshavarz-Haddad, V. Ribeiro, and R. Riedi. Broadcast capacity in multihop wireless networks. In ACM MobiCom'06, pages 239-250, 2006.

[10] A. Keshavarz-Haddad and R. Riedi. On the broadcast capacity of multihop wireless networks: Interplay of power, density and interference. In 4th Annual IEEE Communications Society Conference on Sensor, Mesh and Ad Hoc Communications and Networks (SECON), 2007.

[11] M. Kodialam and T. Nandagopal. Characterizing achievable rates in multi-hop wireless networks: the joint routing and scheduling problem. In ACM MobiCom '03, pages 42-54, 2003.

[12] S. Kulkarni and P. Viswanath. A deterministic approach to throughput scaling in wireless networks. Information Theory, IEEE Transactions on, 50(6):1041-1049, 2004.

[13] P. Kyasanur and N. H. Vaidya. Capacity of multi-channel wireless networks: impact of number of channels and interfaces. In $A C M$ MobiCom '05, pages 43-57, 2005.

[14] J. Li, C. Blake, D. S. J. D. Couto, H. I. Lee, and R. Morris. Capacity of ad hoc wireless networks. In ACM MobiCom, 2001.

[15] X.-Y. LI, S.-J. TANG, AND O. Frieder. Multicast capacity for large scale wireless ad hoc networks. In ACM Mobicom (2007).

[16] M. Penrose. The longest edge of the random minimal spanning tree. Annals of Applied Probability, 7:340-361, 1997.

[17] P. Santi and D. M. Blough. The critical transmitting range for connectivity in sparse wireless ad hoc networks. IEEE Trans. on Mobile Computing, 2:25-39, Mar. 2003.

[18] S. Shakkottai, X. Liu, and R. Srikant. The multicast capacity of ad hoc networks. In ACM Mobihoc, 2007.

[19] J. M. Steele. Growth rates of euclidean minimal spanning trees with power weighted edges. The Annals of Probability, 16(4):1767-1787, Oct 1988.

[20] B. Tavli. Broadcast capacity of wireless networks. IEEE Communication Letters, 10(2), February 2006.

[21] V. Vapnik and A. Chervonenkis. On the uniform convergence of relative frequencies of events to their probabilities. Theory of Probability and its Applications, 16(2):264-280, 1971

[22] P.-J. Wan, K. M. Alzoubi, and O. Frieder. Distributed construction of connected dominating set in wireless ad hoc networks. In INFOCOM, 2002.

[23] W. Wang, Y. Wang, X.-Y. Li, W.-Z. Song, and O. Frieder. Efficient interference aware TDMA link scheduling for static wireless mesh networks. In ACM MobiCom, 2006.

\section{APPENDiX}

In this appendix, we will show that the $\mathrm{VC}$-dimension of $\mathcal{F}$ is $d=\Theta(\log k)$.

To prove this, we first study the VC-dimension of the following system. Let $X$ be the universal set of 2-dimensional segments. For an axis-aligned square $h$ with a fixed sidelength, let $X(h)$ be the set of all segments from $X$ that intersect (or is contained inside) the square $h$. Let

$$
\mathcal{S}=\left\{X(h) \mid h \text { is an axis-aligned square with size } \frac{r}{\sqrt{5}}\right\} .
$$

Given any set of $m$ line segments $\mathcal{L}=\left\{L_{1}, L_{2}, \cdots, L_{m}\right\}$, we show that the cardinality of

$$
\Pi_{\mathcal{S}}(\mathcal{L})=\left\{\mathcal{L}(h) \mid h \text { is a } 2 \mathrm{D} \text { square with side-length } \frac{r}{\sqrt{5}}\right\}
$$

is polynomial of $m$.

Lemma 30: The cardinality of $\Pi_{\mathcal{S}}(\mathcal{L})$ is at most $2 m^{2}$, where $m$ is the cardinality of $\mathcal{L}$.

Proof: This is essentially to study the number of different sets of segments that can be intersected by all 2-dimensional solid square $h$. Imagine that we move a square $J$ (with fixed side-length) all over the 2-dimensional space and at any 
moment we can only see the region not covered by the square $J$. A view is defined as the set of (partial or full) segments that can be seen through the square $J$. Then the view will change only if one the following 4 events happens:

1) A segment starts entering the view and the first point seen is its end-point.

2) A segment starts entering the view and the first point seen is an interior point of the segment.

3) A segment starts leaving the view and the last point seen is its end-point.

4) A segment starts leaving the view and the last point seen is an interior point of the segment.

For scenarios 1) and 3), the square $J$ must have one of its sideedge touching an end-point of some segment. For scenarios 2 ) and 4), it must be the case that a corner of the square $J$ touches the segment on that interior point. See Figure 3 for illustration. When one of the above 4 events happens to the

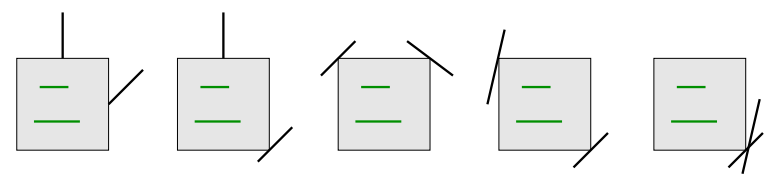

Fig. 3. Scenarios when a square is stable.

square $J$, we call that $J$ has a support. Obviously, when a square has only one support, we can still move the square without changing the view, while keep this support. We can keep moving until the square has another support. A square (axis-aligned and with a fixed side-length) is called stable if it has at least 2 supports (at least one at $x$-axis and one at $y$-axis). Notice that there is a degenerate case here: we cannot find another support when we move a square to find another support. In this degenerated case, the square is called stable if one of its corners touches an end-point of a segment. Thus, the cardinality of $\Pi_{\mathcal{S}}(\mathcal{L})$ is at most the number of stable squares produced by these segments. Notice that, given any pair of segments, it can produce at most 4 stable squares. See Figure 4 for illustration. Thus, the cardinality of $\Pi_{\mathcal{S}}(\mathcal{L})$ is at most

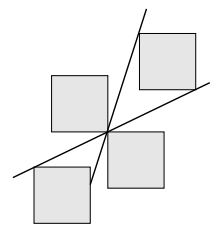

(a)

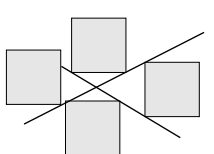

(b) (c)

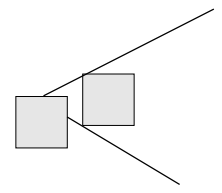

Fig. 4. Scenarios when two segments can produce a stable square.

$\frac{m^{2}}{2} \cdot 4=2 m^{2}$. This finishes the proof.

Notice that, for $m$ segments $\mathcal{L}$, the cardinality of $\Pi_{\mathcal{S}}(\mathcal{L})$ is at most $\frac{m^{2}}{2} \cdot 4=2 m^{2}$. It implies that when a set $\mathcal{L}$ with cardinality $m$ is shatterable by $\mathcal{S}, 2 m^{2} \geq 2^{m}$. Thus, $m<7$. Thus, the VC-dimension of $\mathcal{S}$ is at most 6 .

Lemma 31: The VC-dimension of $\mathcal{F}$ is $O(\log k)$.

Proof: Consider any set $\mathcal{T}=\left\{T_{1}, T_{2}, \cdots, T_{m-1}, T_{m}\right\}$ that is shatterable by $\mathcal{F}$. If we consider only the segments in these trees, there are $m(k-1)$ segments. From Lemma 30, we know that the cardinality of $\Pi_{\mathcal{F}}(\mathcal{T})$ is at most $2 m^{2} \cdot(k-1)^{2}$. Thus, if $\mathcal{T}$ is shatterable by $\mathcal{F}$, we have

$$
2 m^{2} \cdot(k-1)^{2} \geq 2^{m}
$$

Thus, $m<3 \log k$, when $k \geq 2^{12}$. When $k<2^{12}, m$ is also at most a constant. This finishes the proof.

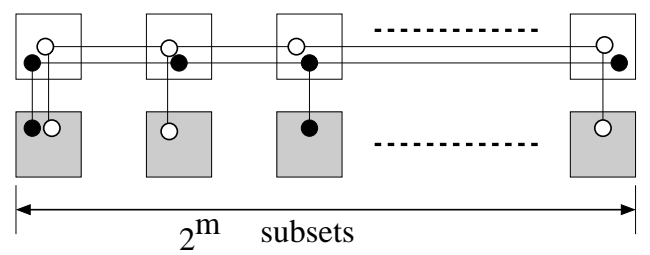

Fig. 5. An example that VC-dimension of trees is $\Theta(\log k)$. Here shaded squares will produce $2^{m}$ distinctive subsets. Here white nodes belong to one tree, and black nodes belong to another tree.

Lemma 32: The VC-dimension of $\mathcal{F}$ is $\Omega(\log k)$.

Proof: We show by example that the VC-dimension of $\mathcal{F}$ is at least $\log \frac{2 k}{3}$. See Figure 5 for illustration. In the example, we will present $m$ trees such that we can view exactly any subset of these $m$ trees using a shaded square with a fixed side-length $\ell$. There are $2^{m}$ such subsets. We will have $2^{m}$ disjoint shaded squares, each corresponding to a subset. Every tree will put one terminal in a shaded square if it belongs to the corresponding subset. Thus, we need exactly $2^{m-1}$ terminals from each tree that will be put in these shaded squares. To make sure that trees generated will not cross a shaded square which it does not belong to, we will an add additional node on top of each shaded square: in the spanning tree, a node in the shaded square will only connect with nodes in the unshaded squares. Then it is easy to see that the total number of nodes a tree needed is $2^{m}+2^{m-1}$ (each for $2^{m}$ squares on top and each for $2^{m-1}$ squares on bottom). Thus, we set $m$ as $2^{m+1}+2^{m}>$ $k \geq 2^{m}+2^{m-1}$. This implies that $\log \frac{2 k}{3}-1<m \leq \log \frac{2 k}{3}$. The theorem then follows.

Thus, the VC-dimension of $\mathcal{F}$ is $d=\Theta(\log k)$. The above theorem also implies that the VC-dimension of any set of trees with at most $n$ edges is at most $O(\log n)$.

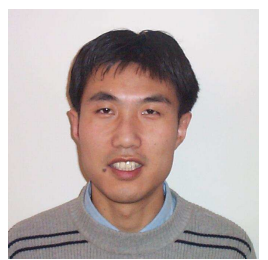

Xiang-Yang Li received MS (2000) and PhD (2001) degree at Department of Computer Science from University of Illinois at Urbana-Champaign. He received his Bachelor degree at Department of Computer Science and Bachelor degree at Department of Business Management from Tsinghua University, P.R. China, both in 1995.

He has been with Department of Computer Science at the Illinois Institute of Technology since 2000. Currently he is an Associate Professor of Department of Computer Science, IIT. He also holds visiting professorship or adjunct-professorship at the following universities: TianJin University, WuHan University, NanJing University and Microsoft Research Asia. His research interests span the wireless ad hoc networks, computational geometry, game theory, and cryptography and network security. $\mathrm{He}$ served various positions (such as conference chair, local arrangement chair) at numerous international conferences. $\mathrm{He}$ is an editor of $\mathrm{Ad} \mathrm{Hoc} \&$ Sensor Wireless Networks: An International Journal. He recently also coorganized a special issue of ACM MONET on non-cooperative computing in wireless networks and a special issue of IEEE Journal of Selected Area in Communications. 